\title{
DYNAMICS OF THE SPATIALLY HOMOGENEOUS BIANCHI TYPE I EINSTEIN-VLASOV EQUATIONS
}

\author{
J. MARK HeinZLE* \\ Max Planck Institute for Gravitational Physics, \\ Albert Einstein Institute, \\ Am Mühlenberg 1, D-14476 Golm, Germany \\ Claes UgGla ${ }^{\dagger}$ \\ Department of Physics, \\ University of Karlstad, S-651 88 Karlstad, Sweden
}

\begin{abstract}
We investigate the dynamics of spatially homogeneous solutions of the EinsteinVlasov equations with Bianchi type I symmetry by using dynamical systems methods. All models are forever expanding and isotropize toward the future; toward the past there exists a singularity. We identify and describe all possible past asymptotic states; in particular, on the past attractor set we establish the existence of a heteroclinic network, which is a new type of feature in general relativity. This illustrates among other things that Vlasov matter can lead to quite different dynamics of cosmological models as compared to perfect fluids.
\end{abstract}

PACS numbers: 04.20.Dw, 98.80.Bp

\footnotetext{
*Electronic address: Mark.Heinzle@aei.mpg.de
}

${ }^{\dagger}$ Electronic address: claes.uggla@kau.se 


\section{Introduction}

In general relativity and cosmology, our knowledge about spatially homogeneous cosmological models has increased substantially over the years, and we are able to say that, for a large number of models, the qualitative behaviour of solutions is now well understood; see [1] for an overview. The majority of results, however, concerns solutions of the Einstein equations coupled to a perfect fluid (usually with a linear equation of state). It is thus important to note that these results are in general not robust, i.e., not structurally stable, under a change of the matter model; significant changes of the qualitative behaviour of solutions occur, for instance, for collisionless matter.

Several fundamental results on spatially homogeneous diagonal models of Bianchi type I with collisionless matter have been obtained in 2. Diagonal locally rotationally symmetric (LRS) models have been investigated successfully by using dynamical systems methods, see [3] for the case of massless particles, and [4, 5] for the massive case. In particular, solutions have been found whose qualitative behaviour is different from that of any perfect fluid model of the same Bianchi type.

The purpose of this article is to re-investigate the diagonal (non-LRS) Bianchi type I models with collisionless matter. Our analysis is based on dynamical systems techniques, which enables us to obtain a more detailed picture of the global dynamics than the one previously given. In particular, we show that the dynamical behaviour toward the past singularity of the collisionless matter model differs considerably from the Bianchi type I perfect fluid model.

The outline of the paper is as follows. In Section 2 we reformulate Einstein's field equations for the diagonal Bianchi type I case with collisionless matter as a reduced dimensionless dynamical system on a compact state space. In Section 3 we give the fixed points of the system and list and discuss a hierarchy of invariant subsets of the state space, which is associated with a hierarchy of monotone functions. In Section 4 we first present the results of the local dynamical systems analysis; subsequently, we focus on the analysis of the global dynamics: we establish two theorems that formulate the future and past asymptotic behaviour of solutions, respectively. As regards the future asymptotics we show that all models isotropize asymptotically toward the future. The past asymptotic behaviour is more complicated since there exists several types of past asymptotic behaviour; in particular we establish that the past attractive set resides on a set that contains a socalled heteroclinic network. The proof of the theorems is based on methods from global dynamical systems analysis; in particular, we exploit the hierarchy of monotone functions in conjunction with the monotonicity principle. Finally, in Section [5] we conclude with some remarks about our results and their implications. Appendix $\mathrm{A}$ provides a brief introduction to relevant background material from the theory of dynamical systems; in particular we cite the monotonicity principle. The proofs of some of the statements in the main text are given in Appendix B and $\mathrm{C}$. In Appendix D we discuss the physical interpretation of one of the most important boundaries of our state space formulation. 


\section{The reflection-symmetric Bianchi type I Einstein-Vlasov system}

In a spacetime with Bianchi type I symmetry the spacetime metric can be written as

$$
d s^{2}=-d t^{2}+g_{i j}(t) d x^{i} d x^{j}, \quad i, j=1,2,3,
$$

where $g_{i j}$ is the induced Riemannian metric on the spatially homogeneous surfaces $t=$ const. Since the metric is constant on $t=$ const, it follows that the Ricci tensor of $g_{i j}$ vanishes. Einstein's equations, in units $G=1=c$, decompose into the evolution equations,

$$
\partial_{t} g_{i j}=-2 k_{i j}, \quad \partial_{t} k^{i}{ }_{j}=\operatorname{tr} k k^{i}{ }_{j}-8 \pi T^{i}{ }_{j}+4 \pi \delta^{i}{ }_{j}\left(T_{k}^{k}-\rho\right)-\Lambda \delta^{i}{ }_{j},
$$

and the Hamiltonian and momentum constraint

$$
(\operatorname{tr} k)^{2}-k_{j}^{i} k_{i}^{j}-16 \pi \rho-2 \Lambda=0, \quad j_{k}=0 .
$$

Here, $k_{i j}$ denotes the second fundamental form of the surfaces $t=$ const. The matter variables are defined as components of the energy-momentum tensor $T_{\mu \nu}(\mu=0,1,2,3)$, according to $\rho=T_{00}, j_{k}=T_{0 k} ; T_{i j}$ denotes the spatial components. The cosmological constant $\Lambda$ is set to zero in the following; the treatment of the case $\Lambda>0$ is straightforward once the case $\Lambda=0$ has been solved, cf. the remarks in the conclusions.

In this paper we consider collisionless matter (Vlasov matter), i.e., an ensemble of freely moving particles described by a non-negative distribution function $f$ defined on the mass shell bundle $P M \subseteq T M$ of the spacetime; for simplicity we consider particles with equal mass $m$. The spacetime coordinates $\left(t, x^{i}\right)$ and the spatial components $v^{i}$ of the fourmomentum $v^{\mu}$ (measured w.r.t. $\partial / \partial x^{\mu}$ ) provide local coordinates on $P M$ so that $f=$ $f\left(t, x^{i}, v^{j}\right)$. Compatibility with Bianchi type I symmetry forces the distribution function $f$ to be homogeneous, i.e., $f=f\left(t, v^{j}\right)$. The evolution equation for $f$ is the Vlasov equation (the Liouville equation)

$$
\partial_{t} f+\frac{v^{j}}{v^{0}} \partial_{x^{j}} f-\frac{1}{v^{0}} \Gamma_{\mu \nu}^{j} v^{\mu} v^{\nu} \partial_{v^{j}} f=\partial_{t} f+2 k_{l}^{j} v^{l} \partial_{v^{j}} f=0 .
$$

The energy-momentum tensor associated with the distribution function $f$ is given by

$$
T^{\mu \nu}=\int f v^{\mu} v^{\nu} \operatorname{vol}_{P M}
$$

where $\operatorname{vol}_{P M}=(\operatorname{det} g)^{1 / 2} v_{0}^{-1} d v^{1} d v^{2} d v^{3}$ is the induced volume form on the mass shell; $v_{0}$ is understood as a function of the spatial components, i.e., $v_{0}^{2}=m^{2}+g_{i j} v^{i} v^{j}$. The components $\rho, j_{k}$, and $T_{i j}$, which enter in (2a) and (2b) can thus be written as

$$
\begin{aligned}
\rho & =\int f\left(m^{2}+g^{i j} v_{i} v_{j}\right)^{1 / 2}(\operatorname{det} g)^{-1 / 2} d v_{1} d v_{2} d v_{3}, \\
j_{k} & =\int f v_{k}(\operatorname{det} g)^{-1 / 2} d v_{1} d v_{2} d v_{3}, \\
T_{i j} & =\int f v_{i} v_{j}\left(m^{2}+g^{k l} v_{k} v_{l}\right)^{-1 / 2}(\operatorname{det} g)^{-1 / 2} d v_{1} d v_{2} d v_{3} .
\end{aligned}
$$


The Einstein-Vlasov system (2) is usually considered for particles of mass $m>0$, however, the system also describes massless particles if we set $m=0$. (For a detailed introduction to the Einstein-Vlasov system we refer to [8] and 9].)

The general spatially homogeneous solution of the Vlasov equation (2C) in Bianchi type I is

$$
f\left(t, v^{i}\right)=f_{0}\left(v_{i}\right),
$$

where the $v_{i}$ are the covariant components of the momenta and $f_{0}$ is an arbitrary nonnegative function, see [10]. (By inserting (3) into (2C) and using that $v_{i}=g_{i j}(t) v^{j}$ it is easy to check that $f_{0}\left(v_{i}\right)$ is a solution.) The momentum constraint in (2b) then reads

$$
\int f_{0}\left(v_{i}\right) v_{k} d v_{1} d v_{2} d v_{3}=0
$$

Henceforth, for simplicity, $f_{0}$ is assumed to be compactly supported.

There exists a subclass of Bianchi type I Einstein-Vlasov models that is naturally associated with the constraint (4): the class of "reflection-symmetric" (or "diagonal") models. The following symmetry conditions are imposed on the initial data:

$$
\begin{gathered}
f_{0}\left(v_{1}, v_{2}, v_{3}\right)=f_{0}\left(-v_{1},-v_{2}, v_{3}\right)=f_{0}\left(-v_{1}, v_{2},-v_{3}\right)=f_{0}\left(v_{1},-v_{2},-v_{3}\right), \\
g_{i j}\left(t_{0}\right), k_{i j}\left(t_{0}\right) \text { diagonal } .
\end{gathered}
$$

These conditions ensure that $T_{i j}\left(t_{0}\right)$ is diagonal, hence $g_{i j}, k_{i j}$, and $T_{i j}$ are diagonal for all times by (2). In the present paper, we will be concerned with this class of reflectionsymmetric models.

The Einstein-Vlasov system (2) thus reduces to a system for six unknowns, the diagonal components of the metric $g_{i i}(t)$ and the second fundamental form $k_{i}^{i}(t)$ (no summation). The equations are (2a) and the Hamiltonian constraint in (2b). The initial data consists of $g_{i i}\left(t_{0}\right), k_{i}^{i}\left(t_{0}\right)$; in addition we prescribe a distribution function $f_{0}\left(v_{i}\right)$ that provides the source terms in the equations via (2d) and (2f).

In the following we reformulate the Einstein-Vlasov system as a dimensionless system on a compact state space. To that end we introduce new variables and modified matter quantities. Let

$$
H:=-\frac{\operatorname{tr} k}{3}, \quad x:=\sum_{i} g^{i i},
$$

and define the dimensionless variables

$$
\begin{aligned}
& s_{i}:=\frac{g^{i i}}{x}, \quad \Sigma_{i}:=-\frac{k_{i}^{i}}{H}-1, \quad z:=\frac{m^{2}}{m^{2}+x}, \\
& \text { where } \quad \sum_{i} s_{i}=1, \quad \sum_{i} \Sigma_{i}=0 \text {. }
\end{aligned}
$$

The transformation from the variables $\left(g_{i i}, k_{i}^{i}\right)$ to $\left(s_{i}, \Sigma_{i}, x, H\right)$, where $\left(s_{i}, \Sigma_{i}\right)$ are subject to the above constraints, is one-to-one. (Note that $x$ can be obtained from $z$ when $m>0$.) 
By distinguishing one direction $(1,2$, or 3$)$, one can decompose the $s_{i}$ and simultaneously introduce a trace-free adaption of the shear to new $\Sigma_{ \pm}$variables as is done in, e.g., [1] however, since Bianchi type I does not have a preferred direction we will refrain from doing so here.

Next we replace the matter quantities $\rho, T_{i}^{i}$ (no summation) by the dimensionless quantities

$$
\Omega:=\frac{8 \pi \rho}{3 H^{2}}, \quad w_{i}:=\frac{T_{i}^{i}}{\rho}, \quad w:=\frac{1}{3} \sum_{i} w_{i}=\frac{1}{3} \frac{\sum_{i} T_{i}^{i}}{\rho} .
$$

Expressed in the new variables, $w_{i}$ can be written as

$$
w_{i}=\frac{(1-z) s_{i} \int f_{0} v_{i}^{2}\left[z+(1-z) \sum_{k} s_{k} v_{k}^{2}\right]^{-1 / 2} d v_{1} d v_{2} d v_{3}}{\int f_{0}\left[z+(1-z) \sum_{k} s_{k} v_{k}^{2}\right]^{1 / 2} d v_{1} d v_{2} d v_{3}} .
$$

Finally, let us introduce a new dimensionless time variable $\tau$ defined by

$$
\partial_{\tau}=H^{-1} \partial_{t}
$$

henceforth a prime denotes differentiation w.r.t. $\tau$.

We now rewrite the Einstein-Vlasov equations as a set of dimensional equations that decouple for dimensional reasons and a reduced system of dimensionless coupled equations on a compact state space. The decoupled dimensional equations are

$$
\begin{aligned}
H^{\prime} & =-3 H\left[1-\frac{\Omega}{2}(1-w)\right] \\
x^{\prime} & =-2 x\left(1+\sum_{k} \Sigma_{k} s_{k}\right) .
\end{aligned}
$$

The reduced dimensionless system consists of the Hamiltonian constraint, cf. (2b),

$$
1-\Sigma^{2}-\Omega=0, \quad \text { where } \quad \Sigma^{2}:=\frac{1}{6} \sum_{k} \Sigma_{k}^{2},
$$

which we use to solve for $\Omega$, and a coupled system of evolution equations

$$
\begin{aligned}
\Sigma_{i}^{\prime} & =-3 \Omega\left[\frac{1}{2}(1-w) \Sigma_{i}-\left(w_{i}-w\right)\right] \\
s_{i}^{\prime} & =-2 s_{i}\left[\Sigma_{i}-\sum_{k} \Sigma_{k} s_{k}\right] \\
z^{\prime} & =2 z(1-z)\left(1+\sum_{k} s_{k} \Sigma_{k}\right) .
\end{aligned}
$$

In the massive case $m>0$ the decoupled equation for $x$ is redundant since the equation for $z$ is equivalent. In the massless case $m=0$ we have $z=0$; hence, although the equation 
for $x$ does not contribute to the dynamics, $x$ is needed in order to reconstruct the spatial metric from the new variables.

The dimensionless dynamical system (13) together with the constraint (12) describes the full dynamics of the Einstein-Vlasov system of Bianchi type I. In the massive case the state space associated with this system is the space of the variables $\left\{\left(\Sigma_{i}, s_{i}, z\right)\right\}$, i.e.,

$$
\mathcal{X}:=\left\{\left(\Sigma_{i}, s_{i}, z\right) \mid\left(\Sigma^{2}<1\right) \wedge\left(s_{i}>0\right) \wedge(0<z<1)\right\},
$$

where the $s_{i}$ and $\Sigma_{i}$ are subject to the constraints $\sum_{k} s_{k}=1, \sum_{k} \Sigma_{k}=0$. (The inequalities for $s_{i}$ and $\Sigma_{i}$ follow from the definition (7a) and the constraint (12), respectively.) The state space $\mathcal{X}$ is thus five-dimensional.

It will turn out eventually that all solutions asymptotically approach the boundaries of $\mathcal{X}: z=0, z=1, s_{i}=0, \Omega=0\left(\Leftrightarrow \Sigma^{2}=1\right)$. This suggests to include these sets in the analysis, whereby we obtain a compact state space $\overline{\mathcal{X}}$.

The equations on the invariant subset $z=0$ of $\overline{\mathcal{X}}$ are identical to the coupled dimensionless system in the case of massless particles $m=0$. We will therefore refer to the subset $z=0$ as the massless subset; it represents the four-dimensional state space for the massless case.

We conclude this section by looking at some variables in more detail. The inequality $\Sigma^{2} \leq 1$ together with the constraint $\sum_{k} \Sigma_{k}=0$ results in $\left|\Sigma_{i}\right| \leq 2$ for all $i$. Note that equality is achieved when $\left(\Sigma_{1}, \Sigma_{2}, \Sigma_{3}\right)=( \pm 2, \mp 1, \mp 1)$ and permutations thereof, cf. Figure 1 . The matter quantities satisfy

$$
0 \leq w \leq \frac{1}{3}, \quad 0 \leq w_{i} \leq 3 w \leq 1 .
$$

The equalities hold at the boundaries of the state space: $w=0$ iff $z=1$, and $w=\frac{1}{3}$ iff $z=0 ; w_{i}=0$ iff $s_{i}=0$, and $w_{i}=3 w$ iff $s_{i}=1$ (provided that $z<1$; for $z=1$, $\left.0=w_{i}=3 w=0\right)$.

There exists a number of useful auxiliary equations that complement the system (13):

$$
\begin{aligned}
& \Omega^{\prime}=\Omega\left[3(1-w) \Sigma^{2}-\sum_{k} w_{k} \Sigma_{k}\right], \\
& \rho^{\prime}=-\rho\left[3(1+w)+\sum_{k} w_{k} \Sigma_{k}\right] \leq-2 \rho .
\end{aligned}
$$

The inequality in (17) follows by using $\Sigma_{i} \geq-2 \forall i$ and (15). This shows that $\rho$ increases monotonically toward the past which yields a matter singularity, i.e., $\rho \rightarrow \infty$ for $\tau \rightarrow$ $-\infty$. It is often beneficial to consider the equations of the original variables as auxiliary equations, e.g., $\left(g^{i i}\right)^{\prime}=-2 g^{i i}\left(1+\Sigma_{i}\right)$.

\section{$3 \quad$ Fixed points, invariant subsets, and monotone functions}

\subsection{Fixed points}

The dynamical system (13) possesses a number of fixed points, all residing on the boundaries of the state space; see Table 1. 


\begin{tabular}{|c|c|}
\hline Fixed point set & defined by \\
\hline $\mathrm{FS}^{1}$ & $z=1, \Sigma_{j}=0 \forall j$ \\
$\mathrm{KC}_{i}^{1}$ & $0 \leq z \leq 1, \Sigma^{2}=1, s_{i}=1, s_{j}=0 \forall j \neq i$ \\
\hline $\mathrm{TS}_{i}$ & $z=0, \Sigma_{j}=0 \forall j, w_{j}=1 / 3 \forall j, s_{i}=0$ \\
\hline $\mathrm{F}^{0}$ & $z=0, s_{i}=0, \Sigma_{i}=-1, \Sigma_{j}=1 / 2=w_{j} \forall j \neq i$ \\
$\mathrm{D}_{i}^{0}$ & $z=0, \Sigma_{i}=-2, \Sigma_{j}=1 \forall j \neq i, s_{i}=0$ \\
$\mathrm{QL}_{i}^{0}$ & $z=0, \Sigma^{2}=1, s_{i}=1, s_{j}=0 \forall j \neq i$ \\
$\mathrm{KC}_{i}^{0}$ & \multicolumn{2}{|}{} \\
\hline
\end{tabular}

Table 1: The fixed point sets. The range of the index $i$ is always $i=1 \ldots 3$. The superscript denotes the value of $z$; the first kernel letter describes the type of fixed point set; if there is no second kernel letter the fixed point set is just a point; if there is a second kernel letter this letter denotes the dimensionality and character of the set $-\mathrm{S}$ refers to surface, $\mathrm{L}$ stands for line, and $\mathrm{C}$ for circle.

$\mathrm{FS}^{1}$ is a surface of fixed points that correspond to the flat isotropic dust solution. The circles $\mathrm{KC}_{i}^{1,0}$ consist of fixed points with constant $\Sigma_{i}$, satisfying $\Sigma^{2}=1, \sum_{k} \Sigma_{k}=0$, see Figure 1 these fixed points correspond to Kasner solutions. The fixed points on $\mathrm{TS}_{i}$ are associated with the Taub representation of the flat Minkowski spacetime. The intersection of $\mathrm{TS}_{i}$ with $(z=0)$ yields a line of fixed points which we denote $\mathrm{TL}_{i}^{0}$. The fixed points on $\mathrm{QL}_{i}^{0}$ correspond to the non-flat LRS Kasner solutions. $\mathrm{F}^{0}$ is a fixed point that corresponds to the flat isotropic radiation solution. In Appendix $\mathrm{B}$ we prove that $\mathrm{F}^{0}$ is well-defined through the equations $w_{1}=w_{2}=w_{3}=1 / 3$ (which are to be solved for $\left.\left(s_{1}, s_{2}, s_{3}\right)\right)$. The location of $\mathrm{F}^{0}$ depends on the chosen distribution function, since the equations $w_{1}=w_{2}=w_{3}=1 / 3$ involve $f_{0}$. Analogously, the equations $w_{j}=1 / 2(\forall j \neq i)$ yield a unique solution, the fixed point $\mathrm{D}_{i}^{0}$; the location of the point $\mathrm{D}_{i}^{0}$ also depends on $f_{0}$. The fixed points $\mathrm{D}_{i}^{0}$ are associated with a scale-invariant LRS solution (related to a distributional $f_{0}$; see Appendix $\mathrm{D}$ for details).

The LRS points on $\mathrm{KC}_{i}^{0}$ play a particularly important role in the following, which motivates that they are given individual names. We denote the three Taub points on $\mathrm{KC}_{i}^{0}$ defined by $\Sigma_{j}=2$ (and thus $\Sigma_{l}=-1 \forall l \neq j$ ) by $\mathrm{T}_{i j}^{0}$, while we denote the three non-flat LRS point on $\mathrm{KC}_{i}^{0}$ given by $\Sigma_{j}=-2$ (and thus $\Sigma_{l}=1 \forall l \neq j$ ) by $\mathrm{Q}_{i j}^{0}$. The Kasner circles $\mathrm{KC}_{j}^{0}$ and $\mathrm{KC}_{k}^{0}$ are connected by the lines $\mathrm{TL}_{i}^{0}$ and $\mathrm{QL}_{i}^{0}$; the end points of the line $\mathrm{TL}_{i}^{0}$ are the Taub points $\mathrm{T}_{j i}^{0}$ and $\mathrm{T}_{k i}^{0}$; analogously, the end points of $\mathrm{QL}_{i}^{0}$ are the points $\mathrm{Q}_{j i}^{0}$ and $\mathrm{Q}_{k i}^{0}$. (Here, $(i, j, k)$ is an arbitrary permutation of $(1,2,3)$.) The remaining points $\mathrm{T}_{l l}^{0}$ and $\mathrm{Q}_{l l}^{0}(l=1 \ldots 3)$ do not lie on any of the fixed point sets $\mathrm{TL}_{i}^{0}$ or $\mathrm{QL}_{i}^{0}$. This fixed point structure is depicted in Figure 2]

\subsection{Invariant subsets and monotone functions}

The dynamical system (13) possesses a hierarchy of invariant subsets and monotone functions. Since this feature of the dynamical system will turn out to be of crucial importance in the analysis of the global dynamics, we give a detailed discussion. 


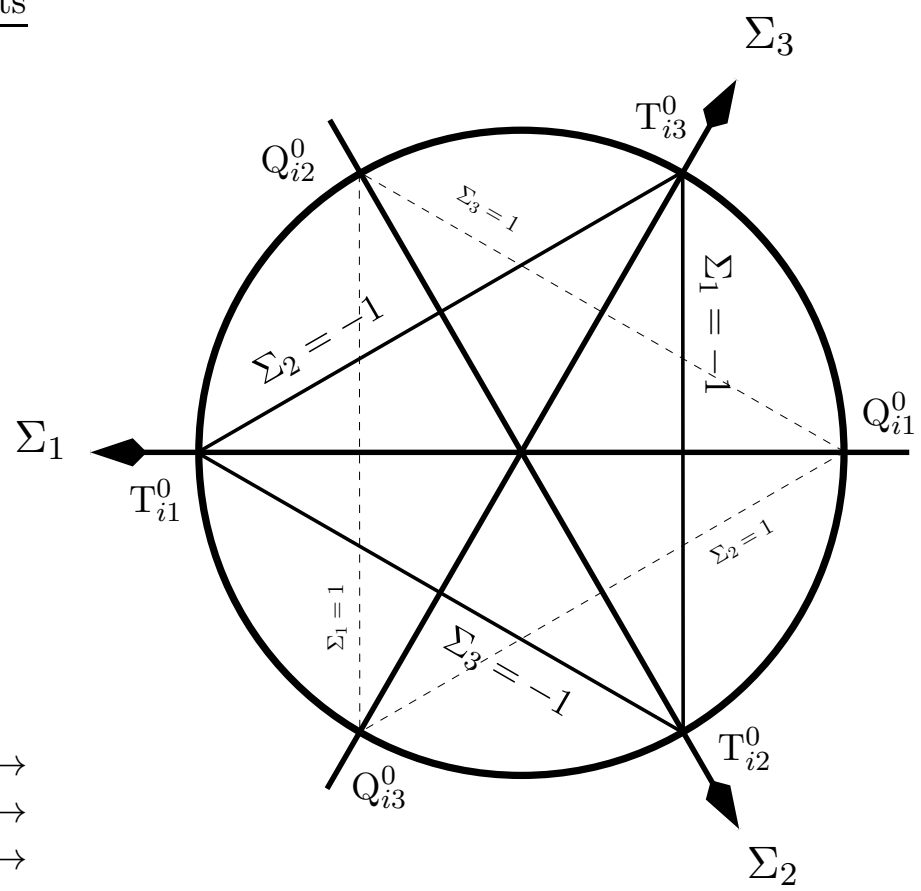

Figure 1: The disc $\Sigma^{2} \leq 1$ and the Kasner circle $\mathrm{KC}_{i}^{0}$.

$\mathcal{X}$ : On the full (interior) state space $\mathcal{X}$ define

$$
M_{(1)}=\left(s_{1} s_{2} s_{3}\right)^{-1 / 3} \frac{z}{1-z} .
$$

A straightforward computation shows

$$
M_{(1)}^{\prime}=2 M_{(1)},
$$

i.e., $M_{(1)}$ is strictly monotonically increasing along orbits in $\mathcal{X}$. Note that $M_{(1)}$ is intimately related to the spatial volume density since $M_{(1)}=m^{2} \operatorname{det}\left(g_{i j}\right)^{1 / 3}$.

$\mathcal{Z}^{1}$ : This subset is characterized by $z=1$. Since $w_{i}=w=0$, the equations for $s_{i}$ decouple, and the essential dynamics is described by the equations $\Sigma_{i}^{\prime}=-(3 / 2)\left(1-\Sigma^{2}\right) \Sigma_{i}$. (Note that these equations are identical to the Bianchi type I equations for dust - it is therefore natural to refer to $\mathcal{Z}^{1}$ as the dust subset.) Explicit solutions for these equations can be obtained by noting that $\Sigma_{1} \propto \Sigma_{2} \propto \Sigma_{3}$ for all solutions, or by using that $\Omega^{\prime}=3 \Sigma^{2} \Omega$.

$\mathcal{Z}^{0}$ : This subset is the massless boundary set $z=0$. Since $w=1 / 3$, the dynamical system (13) reduces to

$$
\Sigma_{i}^{\prime}=-\Omega\left[1+\Sigma_{i}-3 w_{i}\right], \quad s_{i}^{\prime}=-2 s_{i}\left[\Sigma_{i}-\sum_{k} s_{k} \Sigma_{k}\right] .
$$

Consider the function

$$
M_{(2)}=\left(1-\Sigma^{2}\right)^{-1}\left(s_{1} s_{2} s_{3}\right)^{-1 / 6} \int f_{0}\left[\sum_{k} s_{k} v_{k}^{2}\right]^{1 / 2} d v_{1} d v_{2} d v_{3} .
$$




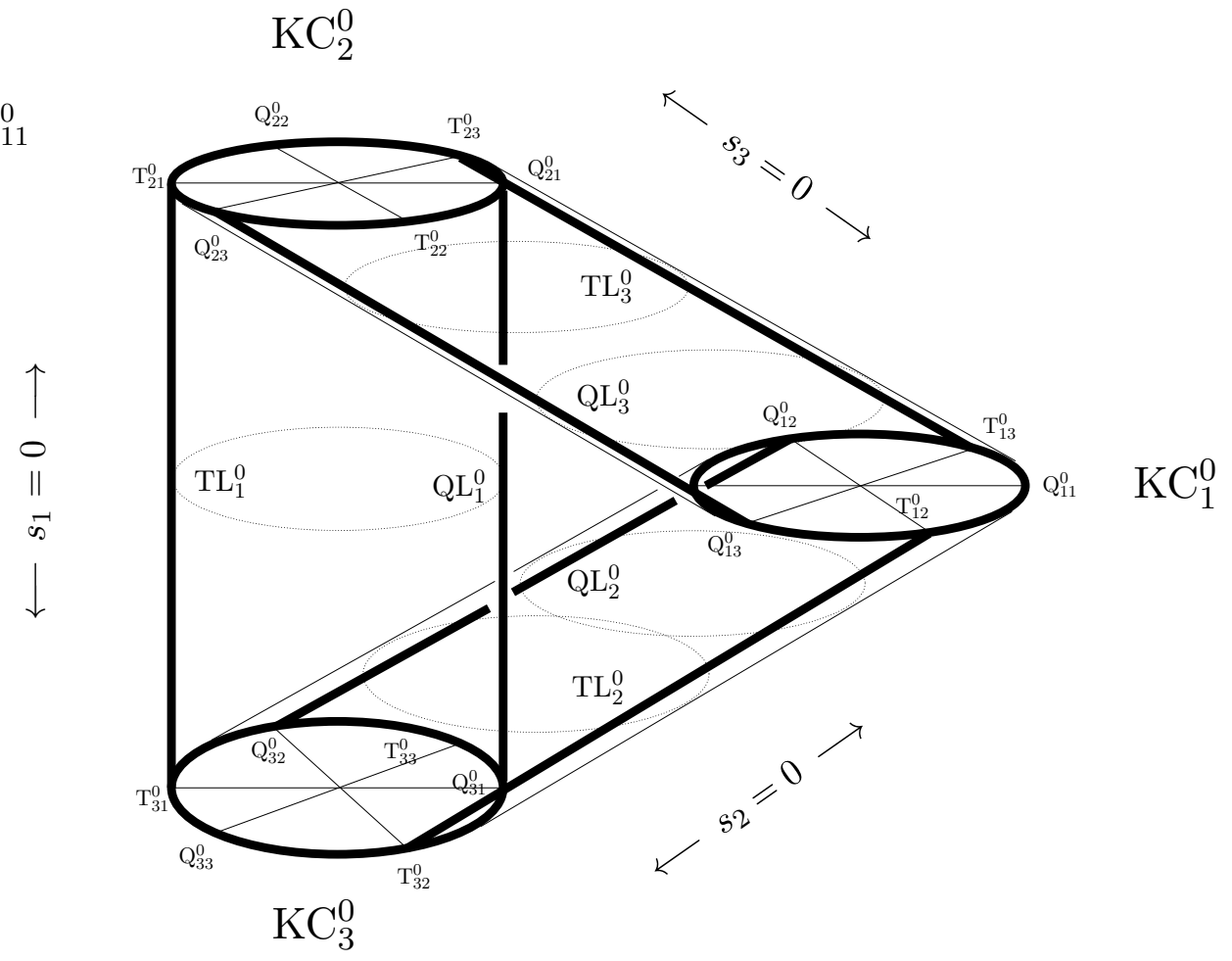

Figure 2: A schematic depiction of the fixed points on $z=0$. The underlying structure is the three sides of the $s_{i}$-triangle $s_{1}+s_{2}+s_{3}=1$ : each point represents a disc $\Sigma^{2} \leq 1$; the vertices contain the Kasner circles $\mathrm{KC}_{i}^{0}$. Bold lines denote the lines of fixed points $\mathrm{TL}_{i}^{0}$, $\mathrm{QL}_{i}^{0}$, and $\mathrm{KC}_{i}^{0}$.

The derivative is

$$
M_{(2)}^{\prime}=-2 \Sigma^{2} M_{(2)},
$$

which yields monotonicity when $\Sigma^{2} \neq 0$. If $\Sigma^{2}=0$, then

$$
M_{(2)}^{\prime}=0, \quad M_{(2)}^{\prime \prime}=0, \quad M_{(2)}^{\prime \prime \prime}=-6 M_{(2)} \sum_{i}\left(w_{i}-\frac{1}{3}\right)^{2} .
$$

Hence, $M_{(2)}$ is strictly monotonically decreasing everywhere on $z=0$, except at the fixed point $\mathrm{F}^{0}$ (for which $\Sigma^{2}=0$ and $w_{1}=w_{2}=w_{3}=1 / 3$ ), where $M_{(2)}$ attains a (positive) minimum. The latter follows from the fact that $\left(1-\Sigma^{2}\right)^{-1}$ is minimal at the point $\Sigma_{i}=0 \forall i$ and that $\partial M_{(2)} / \partial s_{i}=\left(2 s_{i}\right)^{-1}\left[w_{i}-1 / 3\right] M_{(2)}$.

$\mathcal{S}_{i}(i=1,2,3)$ : These invariant boundary subsets are defined by $s_{i}=0$ (which yields $\left.w_{i}=0\right)$. There exists a monotone function on $\mathcal{S}_{1}$,

$$
M_{(3)}=\left(s_{2} s_{3}\right)^{-1 / 2} \frac{z}{1-z}, \quad M_{(3)}^{\prime}=\left(2-\Sigma_{1}\right) M_{(3)} ;
$$

analogous functions can be obtained on $\mathcal{S}_{2}$ and $\mathcal{S}_{3}$ through permutations.

$\mathcal{K}$ : This boundary subset is the vacuum subset defined by $\Omega=0$ (or equivalently $\Sigma^{2}=1$ ). The $\Sigma_{i}$ are constant on this subset, which completely determines the dynamics of the $s_{i}$ 
variables (via (13b) or via the auxiliary equation for $g^{i i}$ ). The Bianchi type I vacuum solution is the familiar Kasner solution and we thus refer to $\mathcal{K}$ as the Kasner subset.

Intersections of the above boundary subsets yield boundary subsets of lower dimensions; those that are relevant for the global dynamics are discussed in the following.

$\mathcal{S}_{i}^{0}$ and $\mathcal{S}_{i}^{1}$ : The intersection between the subset $\mathcal{S}_{i}$ and $\mathcal{Z}^{0}$ and $\mathcal{Z}^{1}$ yields three-dimensional invariant subsets $\left(s_{i}=0\right) \cap(z=0)$ and $\left(s_{i}=0\right) \cap(z=1)$, respectively. On $\mathcal{S}_{i}^{0}$ there exists a monotonically decreasing function:

$$
M_{(4)}=\left(1+\Sigma_{i}\right)^{2}, \quad M_{(4)}^{\prime}=-2 \Omega M_{(4)} .
$$

$\mathcal{S}_{i j}$ : These subsets are defined by setting $s_{i}=0$ and $s_{j}=0(j \neq i)$, i.e., $\mathcal{S}_{i j}=\mathcal{S}_{i} \cap \mathcal{S}_{j}$. On $\mathcal{S}_{i j}$, we have $s_{k}=1(k \neq i, j)$ and $w_{k}=3 w$, because $w_{i}=w_{j}=0$.

$\mathcal{D}_{i}^{0}$ : The subsets $\mathcal{S}_{i}^{0}$ admit two-dimensional invariant subsets $\mathcal{D}_{i}^{0}$ characterized by $(z=$ $0) \cap\left(s_{i}=0\right) \cap\left(\Sigma_{i}=-1\right)$. On $\mathcal{D}_{1}^{0}$ consider the function

$$
M_{(5)}=\left(2+\Sigma_{2} \Sigma_{3}\right)^{-1}\left(s_{2} s_{3}\right)^{-1 / 4} \int f_{0}\left[s_{2} v_{2}^{2}+s_{3} v_{3}^{2}\right]^{1 / 2} d v_{1} d v_{2} d v_{3} ;
$$

analogous functions can be defined on $\mathcal{D}_{2}^{0}$ and $\mathcal{D}_{3}^{0}$. Eqs. (19) imply

$$
M_{(5)}^{\prime}=-\frac{1}{12} M_{(5)}\left[\left(1-2 \Sigma_{2}\right)^{2}+\left(1-2 \Sigma_{3}\right)^{2}\right]
$$

i.e., $M_{(5)}$ is strictly monotonically decreasing unless $\Sigma_{2}=1 / 2=\Sigma_{3}$. In the special case $\Sigma_{2}=1 / 2=\Sigma_{3}$ we obtain

$$
M_{(5)}^{\prime}=0, \quad M_{(5)}^{\prime \prime}=0, \quad M_{(5)}^{\prime \prime \prime}=-\frac{27}{8} M_{(5)}\left[\left(w_{2}-\frac{1}{2}\right)^{2}+\left(w_{3}-\frac{1}{2}\right)^{2}\right] .
$$

Hence, $M_{(5)}$ is strictly monotonically decreasing everywhere on $\mathcal{D}_{1}^{0}$ except for at the fixed point $\mathrm{D}_{1}$, for which $\Sigma_{2}=\Sigma_{3}=w_{2}=w_{3}=\frac{1}{2}$, cf. Table 1. The function $M_{(5)}$ possesses a positive minimum at $D_{1}$. This is because $\left(2+\Sigma_{2} \Sigma_{3}\right)^{-1}$ is minimal at the point $\Sigma_{2}=\Sigma_{3}=$ $1 / 2$ and $\partial M_{(5)} / \partial s_{i}=\left(2 s_{i}\right)^{-1}\left[w_{i}-1 / 2\right] M_{(5)}$ for $i=2,3$.

$\mathcal{K}^{0}$ : The intersection of the Kasner subset $\mathcal{K}=\left(\Sigma^{2}=1\right)$ with the $z=0$ subset yields a 3 -dimensional subset, $\mathcal{K}^{0}$. This subset will play a prominent role in the analysis of the past asymptotic behaviour of solution.

The remaining subsets we consider are not located at the boundaries of the state space, but in the interior; these subsets are invariant under the flow of the dynamical system, if the distribution function $f_{0}$ satisfies certain symmetry conditions.

$\mathrm{LRS}_{i}$ : We define the subset $\mathrm{LRS}_{1}$ of $\mathcal{X}$ through the equations $\Sigma_{2}=\Sigma_{3}, w_{2}=w_{3} ; \mathrm{LRS}_{2,3}$ are defined analogously. In order for these sets to be invariant under the flow of the dynamical system, the distribution function $f_{0}$ must satisfy conditions that ensure compatibility with the LRS symmetry, see Appendix B for details. For an orbit lying on $\mathrm{LRS}_{1}$, Equation (13b) entails that $s_{2}(\tau) \propto s_{3}(\tau)$ (where the proportionality constant exhibits a dependence on $f_{0}$, which enters through the equation $w_{2}=w_{3}$ ), and hence $g_{22} \propto g_{33}$; by rescaling the coordinates one can achieve $g_{22}=g_{33}$, i.e., a line element in an explicit LRS 
form. Hence, the $\mathrm{LRS}_{i}$ subsets, if present as invariant subsets, comprise the solutions with LRS geometry.

FRW: If $f_{0}$ is compatible with an isotropic geometry, see Appendix B for details, the one-dimensional subset characterized by the equations $\Sigma_{i}=0 \forall i$ and $w_{1}=w_{2}=w_{3}=w$ is an invariant subset (in fact: orbit), the FRW subset. The equations $\Sigma_{i}=0$ yield $s_{i}=$ const, whereby we obtain a Friedmann-Robertson-Walker (FRW) geometry, since the spatial coordinates can be rescaled so that $g_{i j} \propto \delta_{i j}$. Note that the location in $s_{i}$ of the FRW subset depends on $f_{0}$, since the equations $w_{1}=w_{2}=w_{3}$, which are to be solved for $\left(s_{1}, s_{2}, s_{3}\right)$, involve $f_{0}$, cf. Appendix B Remarkably, in the massless case the existence of a FRW solution (which corresponds to the fixed point $\mathrm{F}^{0}$ ) does not require any symmetry conditions on $f_{0}$.

\section{Local and global dynamics}

\subsection{Local dynamics}

Let us consider smooth reflection-symmetric Bianchi type I Vlasov solutions that approach fixed point sets when $\tau \rightarrow-\infty$.

Theorem 4.1. In the massive (massless) case there exists

(a) a single orbit that approaches (corresponds to) $\mathrm{F}^{0}$,

(b) three equivalent one-parameter sets of orbits (three single orbits) that approach $\mathrm{D}_{i}^{0}$, $i=1 \ldots 3$,

(c) one three-parameter (two-parameter) set of orbits that approaches $\mathrm{QL}_{1}^{0}$; $\mathrm{QL}_{2}^{0}$ and $\mathrm{QL}_{3}^{0}$ yield equivalent sets,

(d) one four-parameter (three-parameter) set of orbits that approaches the part of $\mathrm{KC}_{1}^{0}$ defined by $1<\Sigma_{1}<2 ; \mathrm{KC}_{2}^{0}$ and $\mathrm{KC}_{3}^{0}$ yield equivalent sets.

Proof. The statements of the theorem follow from the local stability analysis of the fixed point sets $\mathrm{F}^{0}, \mathrm{D}_{i}^{0}, \mathrm{QL}_{i}^{0}, \mathrm{KC}_{i}^{0}$, when combined with the Hartman-Grobman and the reduction theorem, since the fixed points $\mathrm{F}^{0}, \mathrm{D}_{i}^{0}$ are hyperbolic and $\mathrm{QL}_{i}^{0}, \mathrm{KC}_{i}^{0}$ are transversally hyperbolic. This requires the dynamical system to be $\mathcal{C}^{1}$ and this leads to some restrictions on $f_{0}$. However, it is possible to obtain an alternative proof that does not require such restrictions. Such a proof can be obtained from the hierarchical structure of invariant sets; we will refrain from making the details explicit here, since our analysis of the global dynamics below contains all essential ingredients implicitly.

Interpretation of Theorem 4.1] (massive case): A three-parameter set of solutions converges to every non-LRS Kasner solution as $t \rightarrow 0$. (In the state space description three equivalent sets of orbits approach three equivalent transversally stable Kasner arcs that cover all non-LRS Kasner solutions; the equivalence reflects the freedom of permuting the coordinates.) Furthermore, a three-parameter set of solutions approaches the non-flat 
LRS Kasner solution. Hence, in total, a four-parameter set of solutions asymptotically approaches non-flat Kasner states. There exist special solutions with non-Kasner behaviour toward the singularity: one solution isotropizes toward the singularity and a one-parameter set of solutions approaches a non-Kasner LRS solution of the type (46) (three equivalent one-parameter sets of orbits approach three equivalent non-Kasner LRS fixed points associated with this solution). For the latter solutions $\Omega=3 / 4$; these solutions cannot be interpreted as a perfect fluid solutions since they possess anisotropic pressures.

In the following we show that the list of Theorem 4.1 is almost complete: there exist no other attractors toward the singularity with one exception, a heteroclinic network that connects the flat LRS-Kasner points.

\subsection{Global dynamics}

Theorem 4.2. All orbits in the interior of the state space $\mathcal{X}$ of massive particles [state space $\mathcal{Z}^{0}$ of massless particles] converge to $\mathrm{FS}^{1}\left[\mathrm{~F}^{0}\right]$ when $\tau \rightarrow+\infty$; i.e., all smooth reflection-symmetric Bianchi type I Vlasov solutions isotropize toward the future.

A proof of this theorem has been given in [2]. In Appendix [C] we present an alternative proof based on dynamical systems techniques.

Theorem 4.3. The $\alpha$-limit set of an orbit in the interior of the state space is one of the fixed points $\mathrm{F}^{0}, \mathrm{D}_{i}^{0}, \mathrm{QL}_{i}^{0}, \mathrm{KC}_{i}^{0}$, see Theorem [4.1, or it is the heteroclinic network $\mathcal{H}^{0}$. The $\alpha$-limit set of a generic orbit resides on the union of the fixed point sets $\mathrm{KC}_{i}^{0}$ and possibly the heteroclinic network $\mathcal{H}^{0}$.

Remark. A heteroclinic network is defined as a compact connected flow-invariant set that is indecomposable (all points are connected by pseudo-orbits), has a finite nodal set (the set of recurrent points is a finite union of disjoint compact connected flow-invariant subsets), and has finite depth (taking the $\alpha / \omega$-limit iteratively yields the set of recurrent points after a finite number of iterative steps); for details see [1] and references therein. A simple case is a heteroclinic network of depth 1 whose nodes are equilibrium points: it can be regarded as a collection of entangled heteroclinic cycles. The heteroclinic network $\mathcal{H}^{0}$ is of the latter type; it will be introduced in the proof of the theorem.

The remainder of this section is concerned with the proof of Theorem 4.3. The first step in the proof is to gain a detailed understanding of the dynamics on the relevant invariant subspaces of the dynamical system.

\section{Dynamics on $\mathcal{S}_{i}^{0}$}

Lemma 4.4. Consider an orbit in the interior of $\mathcal{S}_{i}^{0}$. Its $\alpha$-limit set is either a fixed point on $\mathrm{KC}_{j}^{0}$ or $\mathrm{KC}_{k}^{0}(i \neq j \neq k), \mathrm{QL}_{i}^{0}$ or $\mathrm{TL}_{i}^{0}$, or it is the heteroclinic cycle $\mathcal{H}_{i}^{0}$. The $\omega$-limit set consists of the fixed point $\mathrm{D}_{i}^{0}$.

Remark. The heteroclinic cycle $\mathcal{H}_{i}^{0}$ will be defined in (26) . 

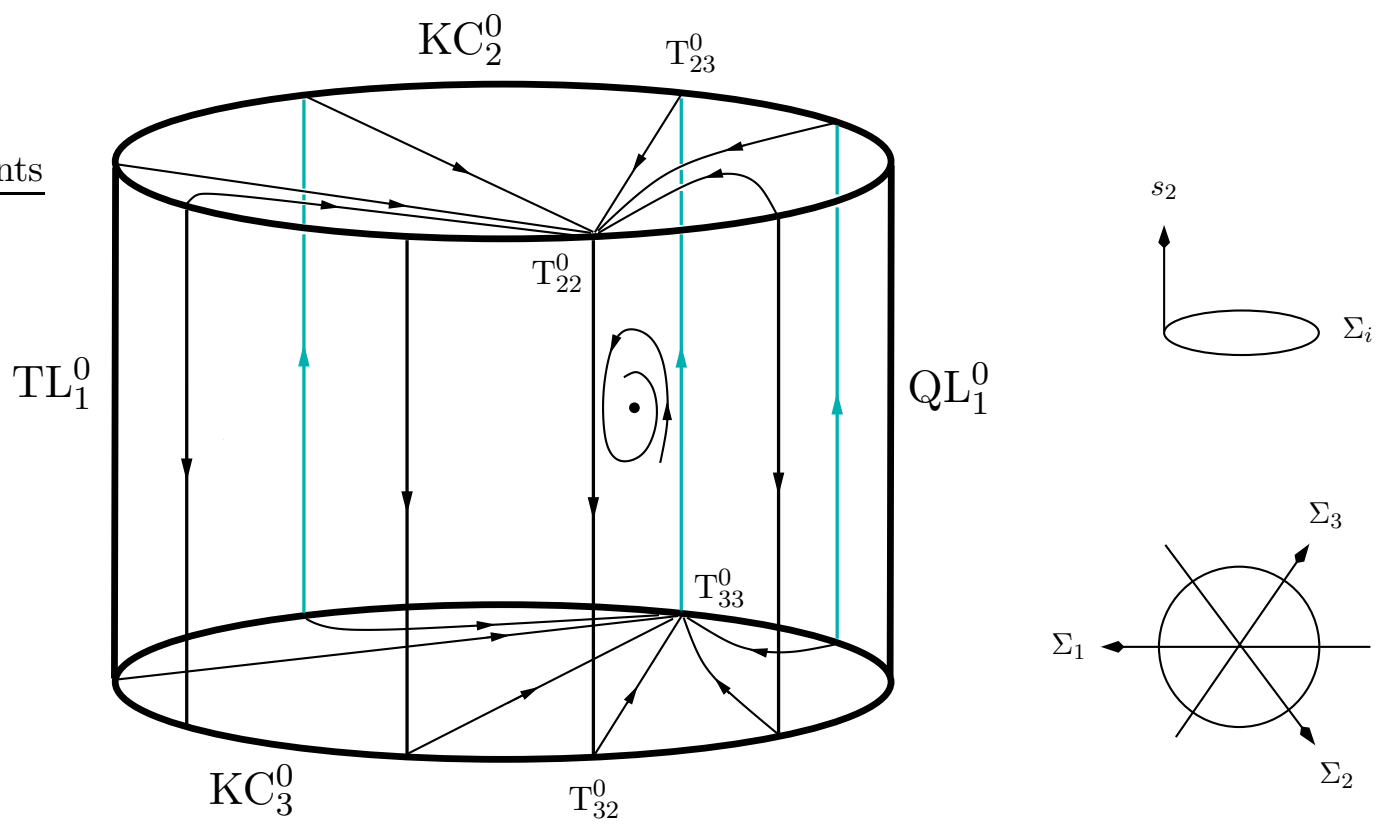

Figure 3: Flow on the boundaries and on the invariant subset $\Sigma_{1}=-1$ on $\mathcal{S}_{1}^{0}$. The fixed point on $\Sigma_{1}=-1$ is the point $\mathrm{D}_{1}^{0}$; the heteroclinic cycle $\mathcal{H}_{1}^{0}$ consists of the fixed points $\mathrm{T}_{22}^{0}, \mathrm{~T}_{32}^{0}, \mathrm{~T}_{33}^{0}, \mathrm{~T}_{23}^{0}$, and the connecting orbits.

Proof. Without loss of generality we consider $\mathcal{S}_{1}^{0}$, which can be described by the variables

$$
0<s_{2}<1\left(s_{3}=1-s_{2}\right) \quad \text { and } \quad \Sigma_{1}, \Sigma_{2}, \Sigma_{3} \quad\left(\sum_{i} \Sigma_{i}=0, \Sigma^{2}<1\right) ;
$$

hence $\mathcal{S}_{1}^{0}$ is represented by the interior of a cylinder, cf. Figure 3. The boundary of $\mathcal{S}_{1}^{0}$ consists of the lateral boundary $\mathcal{S}_{1}^{0} \cap \mathcal{K}^{0}$, the base $\mathcal{S}_{12}^{0}$, and the top surface $\mathcal{S}_{13}^{0}$.

Since $\mathcal{S}_{1}^{0} \cap \mathcal{K}^{0}$ is part of $\mathcal{K}$, it follows that $\Sigma_{i} \equiv$ const for all orbits on $\mathcal{S}_{1}^{0} \cap \mathcal{K}^{0}$. We observe that $s_{2}$ is monotonically increasing (decreasing) when $\Sigma_{2}<\Sigma_{3}\left(\Sigma_{2}>\Sigma_{3}\right)$, since $s_{2}^{\prime}=-2 s_{2}\left(1-s_{2}\right)\left(\Sigma_{2}-\Sigma_{3}\right)$; the two domains are separated by the lines of fixed points $\mathrm{TL}_{1}^{0}$ and $\mathrm{QL}_{1}^{0}$, see Figure 3 .

The key equations to understand the flow on $\mathcal{S}_{12}^{0}$ are

$$
\Omega^{\prime}=\Omega\left(2 \Sigma^{2}-\Sigma_{3}\right) \quad \text { and } \quad \Sigma_{3}^{\prime}=\Omega\left(2-\Sigma_{3}\right) .
$$

From the first equation it follows that all points on $\mathrm{KC}_{3}^{0}$ are transversally hyperbolic repelling fixed points except for $\mathrm{T}_{33}^{0}$; from the second equation we infer that $\mathrm{T}_{33}^{0}$ is the attractor of the entire interior of $\mathcal{S}_{12}^{0}$. Similarly, $\mathrm{T}_{22}^{0}$ is the attractor on $\mathcal{S}_{13}^{0}$, see Figure 3 ,

The plane $\mathcal{D}_{1}^{0}$, defined by $\Sigma_{1}=-1$, is an invariant subset in $\mathcal{S}_{1}^{0}$. In the interior of the plane we find the fixed point $\mathrm{D}_{1}^{0}$; the boundary consists of a heteroclinic cycle $\mathcal{H}_{1}^{0}$,

$$
\mathcal{H}_{1}^{0}: \mathrm{T}_{22}^{0} \rightarrow \mathrm{T}_{32}^{0} \rightarrow \mathrm{T}_{33}^{0} \rightarrow \mathrm{T}_{23}^{0} \rightarrow \mathrm{T}_{22}^{0} .
$$

(Note that analogous cycles $\mathcal{H}_{2}^{0}$ and $\mathcal{H}_{3}^{0}$ exist on $\mathcal{S}_{2}^{0}$ and $\mathcal{S}_{3}^{0}$, respectively.) The function $M_{(5)}$ is monotonically decreasing on $\mathcal{D}_{1}^{0}$, cf. (23). Application of the monotonicity principle, 
see Appendix yields that $\mathrm{D}_{1}^{0}$ is the $\omega$-limit and that $\mathcal{H}_{1}^{0}$ is the $\alpha$-limit for all orbits on $\mathcal{D}_{1}^{0}$, cf. Figure 3 .

Consider now an orbit in $\mathcal{S}_{1}^{0}$ with $\Sigma_{1} \neq-1$. The function $M_{(4)}=\left(1+\Sigma_{1}\right)^{2}$ is monotonically decreasing on $\mathcal{S}_{1}^{0}$, cf. (22). The monotonicity principle implies that the $\omega$-limit lies on $\Sigma_{1}=-1$ or $\Sigma^{2}=1$ (but $\Sigma_{1} \neq \pm 2$ ). Since the logarithmic derivative of $\Omega$ is positive everywhere on $\mathcal{S}_{1}^{0} \cap \mathcal{K}^{0}$ (except at $\mathrm{T}_{22}^{0}$ and $\mathrm{T}_{33}^{0}$ ), i.e., $\left.\Omega^{-1} \Omega^{\prime}\right|_{\Omega=0}=2-\sum_{k} w_{k} \Sigma_{k}>0$, it follows that the "wall" $\mathcal{S}_{1}^{0} \cap \mathcal{K}^{0}$ of the cylinder is repelling everywhere away from $\Sigma_{1}=-1$. Consequently, the $\omega$-limit of the orbit cannot lie on $\Sigma^{2}=1$ but is contained in $\Sigma_{1}=-1$. The fixed point $D_{1}^{0}$ is a hyperbolic sink, as we conclude from the dynamics on $\Sigma_{1}=-1$ and from $\left.\left(1+\Sigma_{1}\right)^{-1}\left(1+\Sigma_{1}\right)^{\prime}\right|_{D_{1}^{0}}=-3 / 4$. Therefore, the a priori possible $\omega$-limit sets on $\Sigma_{1}=-1$ are $\mathrm{D}_{1}^{0}$ and the heteroclinic cycle $\mathcal{H}_{1}^{0}$.

To prove that $\mathrm{D}_{1}^{0}$ is the actual $\omega$-limit we again consider the function $M_{(5)}$. However, we no longer restrict its domain of definition to $\mathcal{D}_{1}^{0}$, but view it as a function on $\mathcal{S}_{1}^{0}$; we obtain

$$
12 M_{(5)}^{\prime}=-M_{(5)}\left[\left(\Sigma_{1}+2 \Sigma_{2}\right)^{2}+\left(\Sigma_{1}+2 \Sigma_{3}\right)^{2}+6\left(\Sigma_{1}+1\right)^{2}-6\left(\Sigma_{1}+1\right)\right] .
$$

The bracket is positive when $\Sigma_{1}<-1$; hence $M_{(5)}$ is decreasing when $\Sigma_{1}<-1$. This prevents orbits with $\Sigma_{1}<-1$ from approaching $\mathcal{H}_{1}^{0}$, since the cycle is characterized by $M_{(5)}=\infty$. Now suppose that there exist an orbit in $\Sigma_{1}>-1$, whose $\omega$-limit is $\mathcal{H}_{1}^{0}$. At late times the trajectory shadows the cycle; hence, for late times, the bracket in (27) is almost always positive along the trajectory - only when the trajectory passes through a small neighbourhood of $\left(\Sigma_{1}, \Sigma_{2}, \Sigma_{3}\right)=(-1,1 / 2,1 / 2)$ the bracket is marginally negative. Since the trajectory spends large amounts of time near the fixed points and the passages from one fixed point to another become shorter and shorter in proportion, it follows that at late times $M_{(5)}$ is decreasing along the orbit (with ever shorter periods of limited increase). This is a contradiction to the assumption that the orbit is attracted by the heteroclinic cycle. We therefore draw the conclusion that $\mathrm{D}_{1}^{0}$ is the global sink on $\mathcal{S}_{1}^{0}$.

Consider again an orbit in $\mathcal{S}_{1}^{0}$ with $\Sigma_{1} \neq-1$. Invoking the monotonicity principle with the function $M_{(4)}$ we find that the $\alpha$-limit of the orbit must be located on $\Sigma^{2}=1, \Sigma_{1} \neq-1$. From the analysis of the flow on the boundaries of the cylinder we obtain that all fixed points on $\Sigma^{2}=1$ except for $\mathrm{T}_{22}^{0}$ and $\mathrm{T}_{33}^{0}$ are transversally hyperbolic. The fixed points on $\mathrm{KC}_{2}^{0}$ with $\Sigma_{2}<\Sigma_{3}$ and the points on $\mathrm{KC}_{3}^{0}$ with $\Sigma_{2}>\Sigma_{3}$ are saddles; the fixed points on $\mathrm{KC}_{2}^{0}$ with $\Sigma_{2}>\Sigma_{3}$ and those on $\mathrm{KC}_{3}^{0}$ with $\Sigma_{2}<\Sigma_{3}$ are transversally hyperbolic sources (except for $\mathrm{T}_{22}^{0}, \mathrm{~T}_{33}^{0}$ ): every point attracts a one-parameter set of orbits from $\mathcal{S}_{1}^{0}$ as $\tau \rightarrow-\infty$. In contrast, each fixed point on $\mathrm{TL}_{1}^{0}$ and $\mathrm{QL}_{1}^{0}$ is a source for exactly one orbit. The structure of the flow on $\Sigma^{2}=1$ implies that the $\alpha$-limit of the orbit in $\mathcal{S}_{1}^{0}$ with $\Sigma_{1} \neq-1$ must be one of the transversally hyperbolic sources.

This establishes Lemma 4.4

\section{Dynamics on $\mathcal{K}^{0}$}

The invariant subset $\mathcal{K}^{0}$ is defined by setting $z=0$ and $\Sigma^{2}=1$; it can be represented by the Cartesian product of the circle $\left(\Sigma^{2}=1\right)$ in the $\Sigma_{i}$-space times the $s_{i}$-triangle given by $\left\{0<s_{1}, s_{2}, s_{3}<1, \sum_{k} s_{k}=1\right\}$. The flow on this space possesses a simple structure: since 

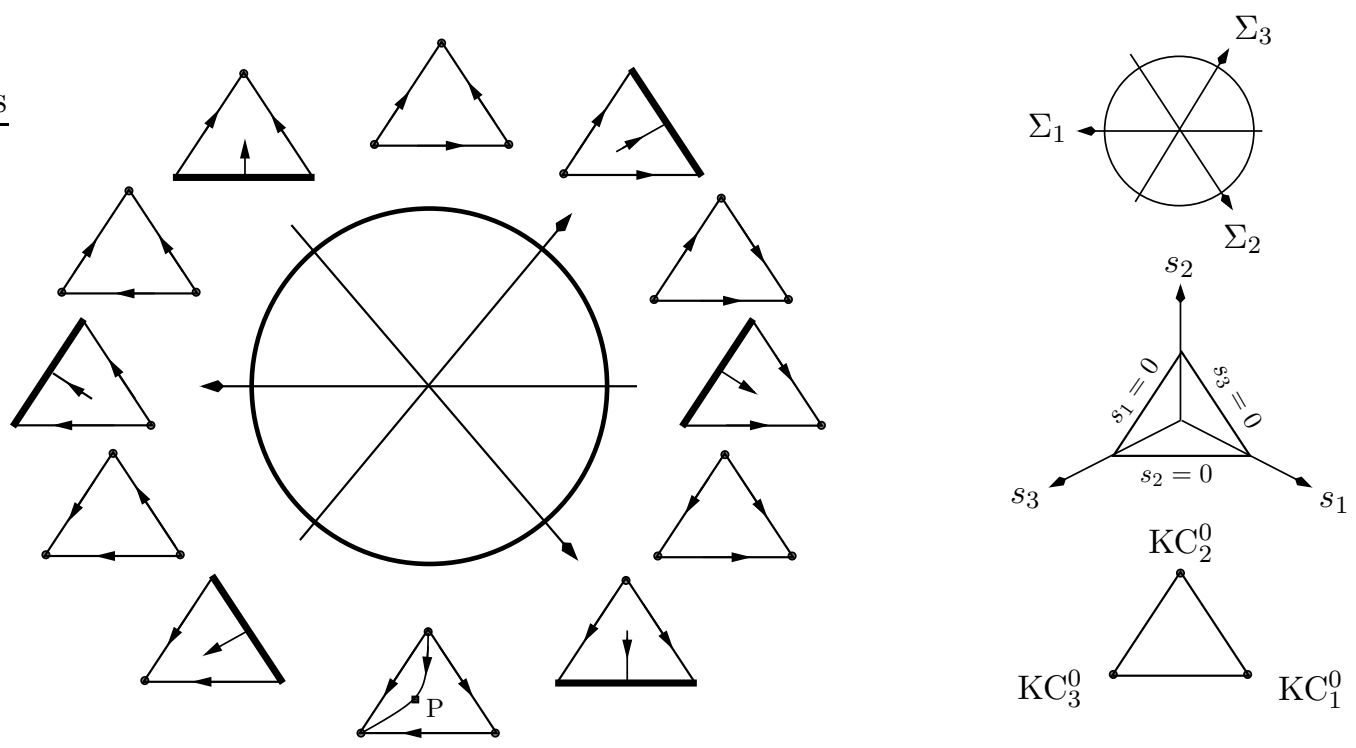

Figure 4: Schematic representation of the flow of the dynamical system on the invariant set $\mathcal{K}^{0}=(z=0) \cap\left(\Sigma^{2}=1\right)$, which is the Cartesian product of the $\left(\Sigma^{2}=1\right)$-circle and the $s_{i}$-triangle. The depicted fixed points are the Kasner fixed points and the lines of fixed points $\mathrm{TL}_{i}^{0}\left(\leftrightarrow \Sigma_{i}=2\right)$ and $\mathrm{QL}_{i}^{0}\left(\leftrightarrow \Sigma_{i}=-2\right)$. All orbits are heteroclinic, like the orbit through the arbitrary point $\mathrm{P}$ that connects $\mathrm{KC}_{2}^{0}$ with $\mathrm{KC}_{3}^{0}$.

$\Sigma_{i}^{\prime} \equiv$ const for all orbits, the dynamical freedom resides in the $s_{i}$-spaces.

A schematic depiction of the flow on $\mathcal{K}^{0}$ is given in Figure 4. All fixed points are located on the boundaries of $\mathcal{K}^{0}$, i.e., on $s_{1}=0, s_{2}=0$, or $s_{3}=0$. The vertices of the $s_{i}$-triangle are the Kasner circles $\mathrm{KC}_{i}^{0}$. If $\left(\Sigma_{1}, \Sigma_{2}, \Sigma_{3}\right) \in\left(\Sigma^{2}=1\right)$ is such that $\Sigma_{k}=2$ (respectively $\Sigma_{k}=-2$ ) for some $k$, then the side $s_{k}=0$ of the triangle is a line of fixed points, $\mathrm{TL}_{k}^{0}$ $\left(\right.$ respectively $\mathrm{QL}_{k}^{0}$ ). Note that all fixed points are transversally hyperbolic on $\mathcal{K}^{0}$, and that they constitute the $\alpha$ - and $\omega$-limit sets for all orbits on $\mathcal{K}^{0}$. The character of the fixed points, i.e., whether they are (transversal) attractors or repellors, depends on the sector of the circle $\left(\Sigma^{2}=1\right)$, see Figure 4

The results about the global dynamics on $\mathcal{S}_{i}^{0}$ and $\mathcal{K}^{0}$ will turn out to be an integral part in the proof of Theorem 4.3. which we will address next. First, we will prove the massless case of the theorem.

\section{Dynamics on $\mathcal{Z}^{0}$}

Let $\gamma$ be an arbitrary orbit in the interior of $\mathcal{Z}^{0}, \gamma \neq\left\{\mathrm{F}^{0}\right\}$. The function $M_{(2)}$ is strictly monotonically decreasing on $\mathcal{Z}^{0}$ (except at $\mathrm{F}^{0}$, where it has a minimum), cf. (20) ff.; hence we can use the monotonicity principle: the $\alpha$-limit set $\alpha(\gamma)$ of $\gamma$ must be located on the boundaries of $\mathcal{Z}^{0}$, i.e., on $\mathcal{S}_{i}^{0}$ or $\mathcal{K}^{0}$. The first step in our analysis is to prove that the interior of the subsets $\mathcal{S}_{i}^{0}$ and $\mathcal{K}^{0}$ cannot belong to $\alpha(\gamma)$, unless $\gamma$ is one of three special orbits. 
Recall from our analysis of $\mathcal{S}_{i}^{0}$ that the fixed point $\mathrm{D}_{i}^{0} \in \mathcal{S}_{i}^{0}$ is a hyperbolic sink on $\mathcal{S}_{i}^{0}$. In the orthogonal direction, however, we obtain $\left.s_{i}^{-1} s_{i}^{\prime}\right|_{\mathrm{D}_{i}^{0}}=3$. It follows that $\mathrm{D}_{i}^{0}$ is a hyperbolic saddle in the state space $\mathcal{Z}^{0}$ and that there exists exactly one orbit $\delta_{i}^{0}$ that emanates from $\mathrm{D}_{i}^{0}$ into the interior of $\mathcal{Z}^{0}$. (Theorem 4.2 implies that $\delta_{i}^{0}$ converges to the global sink $\mathrm{F}^{0}$ as $\tau \rightarrow \infty$.)

Henceforth, let $\gamma$ be different from $\delta_{i}^{0}$. In order to show that $\alpha(\gamma)$ does not contain any point of the interior of $\mathcal{S}_{i}^{0}$, we perform a proof by contradiction: assume that $\alpha(\gamma)$ contains a point $\mathrm{P}$ of the interior of $\mathcal{S}_{i}^{0}$; then the whole orbit through $\mathrm{P}$ and the $\omega$-limit $\omega(\mathrm{P})$ (as well as the $\alpha$-limit) of that orbit must be contained in $\alpha(\gamma)$. As already shown, $\mathrm{D}_{i}^{0}$ is the global attractor on $\mathcal{S}_{i}^{0}$, hence $\alpha(\gamma) \ni \omega(\mathrm{P})=\mathrm{D}_{i}^{0}$. Since the saddle $\mathrm{D}_{i}^{0}$ is in $\alpha(\gamma)$, the unique orbit $\delta_{i}^{0}$ emanating from it is contained in $\alpha(\gamma)$ as well. Thus, ultimately, $\omega\left(\delta_{i}^{0}\right)$, i.e., the point $\mathrm{F}^{0}$, must be contained in $\alpha(\gamma)$; this is a contradiction, since $\mathrm{F}^{0}$ is a sink. Therefore, $\gamma$ cannot contain any $\alpha$-limit point in the interior of $\mathcal{S}_{i}^{0}$. We will now use similar reasoning repeatedly.

Assume next that $\alpha(\gamma)$ contains a point $\mathrm{P}$ in the interior of $\mathcal{K}^{0}$, i.e., a point with $\Sigma^{2}=1$, $0<s_{i}<1 \forall i$. Suppose first that $\Sigma_{k} \neq \pm 2$ for all $k$. Since $\mathrm{P}$ is an element of $\alpha(\gamma)$, the whole orbit through $\mathrm{P}$ and the $\alpha$-limit $\alpha(\mathrm{P})$ of that orbit must be contained in $\alpha(\gamma)$. From the dynamics on $\mathcal{K}^{0}$, cf. Figure 4 it follows that $\alpha(\mathrm{P})$ is one of the Kasner fixed points on $\mathrm{KC}_{i}^{0}$, where $i$ corresponds to the direction determined by $\Sigma_{i}=\max _{k} \Sigma_{k}$; we hence denote $\alpha(\mathrm{P})$ as $\mathrm{K}_{\mathrm{P}}$. Since $\Sigma_{k} \neq \pm 2$, it follows from the previous analysis that $\mathrm{K}_{\mathrm{P}}$ is a transversally hyperbolic source on the subspace $\mathcal{K}^{0} ;\left.\Omega^{-1} \Omega^{\prime}\right|_{\Sigma^{2}=1}=2-\sum_{k} w_{k} \Sigma_{k}>0$ yields that $\mathrm{K}_{\mathrm{P}}$ is a transversally hyperbolic source on the whole space $\mathcal{Z}^{0}$. Since $\alpha(\gamma)$ contains the transversally hyperbolic source $\mathrm{K}_{\mathrm{P}}$, that fixed point necessarily constitutes the entire $\alpha$-limit set, i.e., $\alpha(\gamma)=\mathrm{K}_{\mathrm{P}}$. This is in contradiction to our assumption $\alpha(\gamma) \ni \mathrm{P}$. The omitted cases $\Sigma_{i}= \pm 2$ for some $i$ will be dealt with next.

Suppose that $\Sigma_{i}=-2$ for one index $i$. Assume that $\mathrm{P}$ lies in $\alpha(\gamma)$, therefore $\alpha(\mathrm{P})$ is contained in $\alpha(\gamma)$ as well. The dynamics on $\mathcal{K}^{0}$ implies that $\alpha(\mathrm{P})$ is a fixed point $\mathrm{Q}_{\mathrm{P}}$ on $\mathrm{QL}_{i}^{0}$, cf. Figure 4. This point is a transversally hyperbolic source; $\left.\Omega^{-1} \Omega^{\prime}\right|_{\mathrm{QL}_{i}^{0}}=1$ in this case. By the same argument as above we obtain a contradiction to the assumption $\alpha(\gamma) \ni \mathrm{P}$.

Finally suppose that $\Sigma_{i}=2$ for one index $i$. When we assume that $\mathrm{P}$ is in $\alpha(\gamma)$, then the $\omega$-limit $\omega(\mathrm{P})$ is contained in $\alpha(\gamma)$. From Figure 4 we see that $\omega(\mathrm{P})$ is a fixed point $\mathrm{T}_{\mathrm{P}}$ on $\mathrm{TL}_{i}^{0}$. The point $\mathrm{T}_{\mathrm{P}}$ is a transversally hyperbolic saddle, since $\left.\Omega^{-1} \Omega^{\prime}\right|_{\mathrm{TL}_{i}^{0}}=3$, and there exists exactly one orbit that emanates from it, namely the orbit that connects $\mathrm{T}_{\mathrm{P}}$ with $\mathrm{D}_{i}$ in $\mathcal{S}_{i}^{0}$. Since $\mathrm{T}_{\mathrm{P}} \in \alpha(\gamma)$, that orbit must also be contained in $\alpha(\gamma)$. This is in contradiction to the previous result: $\alpha(\gamma)$ cannot contain interior points of $\mathcal{S}_{i}^{0}$. Hence our assumption $\alpha(\gamma) \ni \mathrm{P}$ was false: the $\alpha$-limit of $\gamma$ cannot contain any interior point of $\mathcal{K}^{0}$.

Our analysis results in the following statement: There exist four special orbits, one trivial orbit corresponding to the fixed point $\mathrm{F}^{0}$ and three orbits, the orbits $\delta_{i}^{0}$, that converge to the fixed points $\mathrm{D}_{i}^{0} \in \mathcal{D}_{i}^{0}$. The $\alpha$-limit set of every orbit $\gamma$ in $\mathcal{Z}^{0}$ different from $\mathrm{F}^{0}$ and $\delta_{i}^{0}$ must be located on the boundaries of the spaces $\mathcal{S}_{i}^{0}$ and $\mathcal{K}^{0}$, i.e., on the union of the boundaries of the cylinders $\mathcal{S}_{i}^{0}$, which we denote by $\partial \mathcal{S}^{0}=\partial \mathcal{S}_{1}^{0} \cup \partial \mathcal{S}_{2}^{0} \cup \partial \mathcal{S}_{3}^{0}$. The set $\partial \mathcal{S}^{0}$ is depicted in Figure 2 it comprises the lateral surfaces of the cylinders and the base/top 


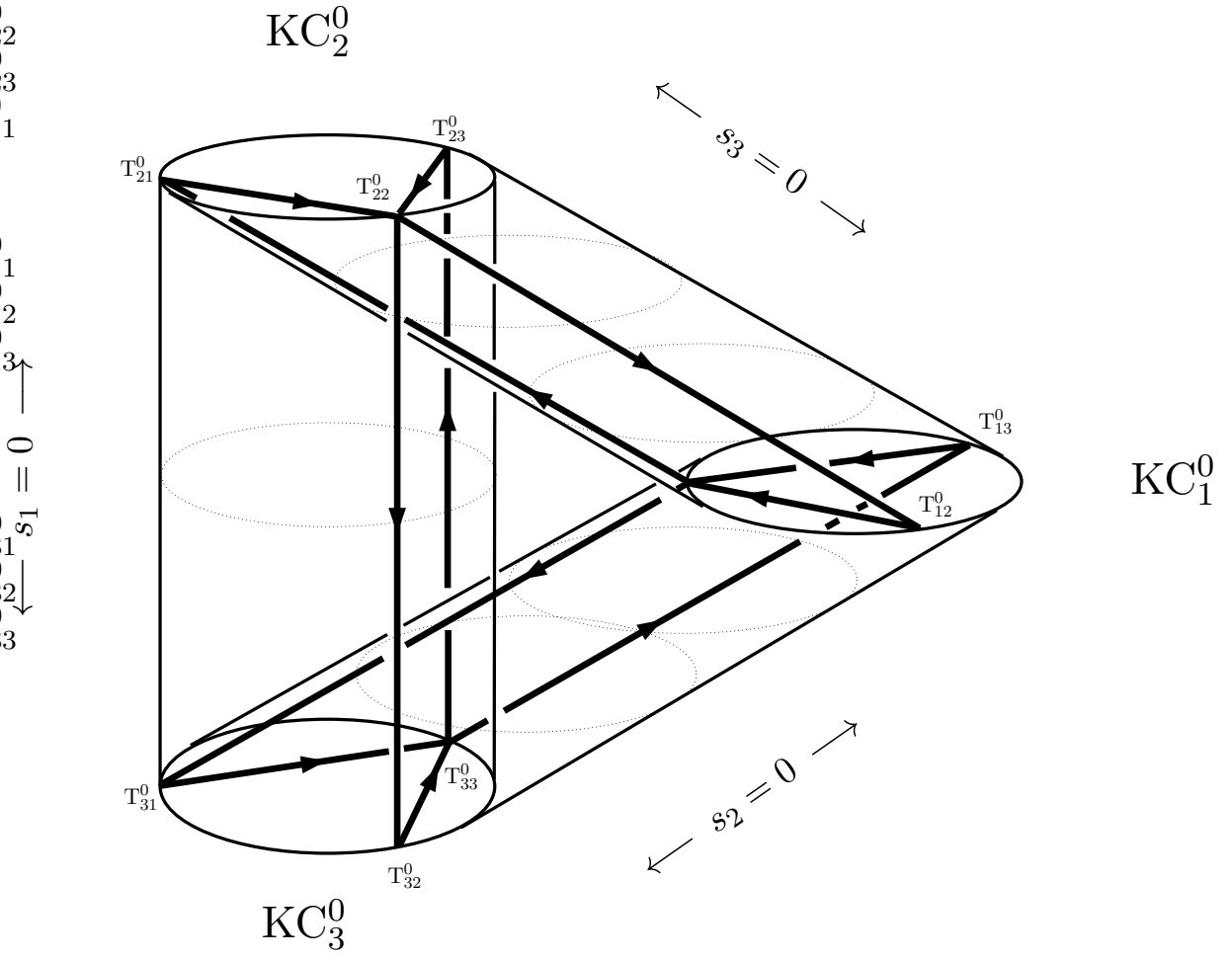

Figure 5: The heteroclinic network $\mathcal{H}^{0}$ that exists on the set $\partial \mathcal{S}^{0}$. Its building blocks are the heteroclinic cycles $\mathcal{H}_{1}^{0}, \mathcal{H}_{2}^{0}, \mathcal{H}_{3}^{0}$.

surfaces.

All fixed points on $\partial \mathcal{S}^{0}$ are transversally hyperbolic except for the points $\mathrm{T}_{i i}^{0}$ : $\mathrm{TL}_{i}^{0}$ consists of transversally hyperbolic saddles; in contrast, the fixed points on $\mathrm{QL}_{i}^{0}$ are transversally hyperbolic sources; points on $\mathrm{KC}_{i}^{0}$ with $\Sigma_{i}>1, \Sigma_{i} \neq 2$ are sources while those with $\Sigma_{i}<1$ are saddles. Combining the analysis of the preceding sections, see Figs. 3 and 4 . we obtain, more specifically: each point on $\mathrm{QL}_{i}^{0}$ is a source for a one-parameter family of orbits that emanate into the interior of $\mathcal{Z}^{0}$, and each point on $\mathrm{KC}_{i}^{0}$ with $\Sigma_{i}>1\left(\Sigma_{i} \neq 2\right)$ is the source for a two-parameter family. (The points with $\Sigma_{i}=1$ on $\mathrm{KC}_{i}^{0}$ are the two points $\mathrm{Q}_{i j}^{0} \in \mathrm{QL}_{j}^{0}$ and $\mathrm{Q}_{i k}^{0} \in \mathrm{QL}_{k}^{0}$. Each of these two points is a transversally hyperbolic source for a one-parameter family of orbits, however, those orbits are not interior orbits, but remain on the boundary of $\mathcal{Z}^{0}$.)

The non-transversally hyperbolic fixed points $\mathrm{T}_{i i}^{0}$ are part of a special structure that is present on $\partial \mathcal{S}^{0}$ : the set $\partial \mathcal{S}^{0}$ exhibits a robust heteroclinic network $\mathcal{H}^{0}$ (of depth 1 ), see, e.g., [1] for a discussion of heteroclinic networks; the network $\mathcal{H}^{0}$ is depicted in Figure [5] a schematic depiction is given in Figure 6. In particular we observe that the heteroclinic cycles $\mathcal{H}_{i}^{0}$ of the spaces $\mathcal{S}_{i}^{0}$ appear as heteroclinic subcycles of the network.

A straightforward analysis of the flow on $\partial \mathcal{S}^{0}$ using the same type of reasoning as above leads to the result that there exist no other structures on $\partial \mathcal{S}^{0}$ that could serve as $\alpha$-limits for an interior $\mathcal{Z}^{0}$-orbit $\gamma$. We have thus proved the following statement: The $\alpha$-limit of $\gamma$ is one of the transversally hyperbolic sources listed above, or it is the heteroclinic 


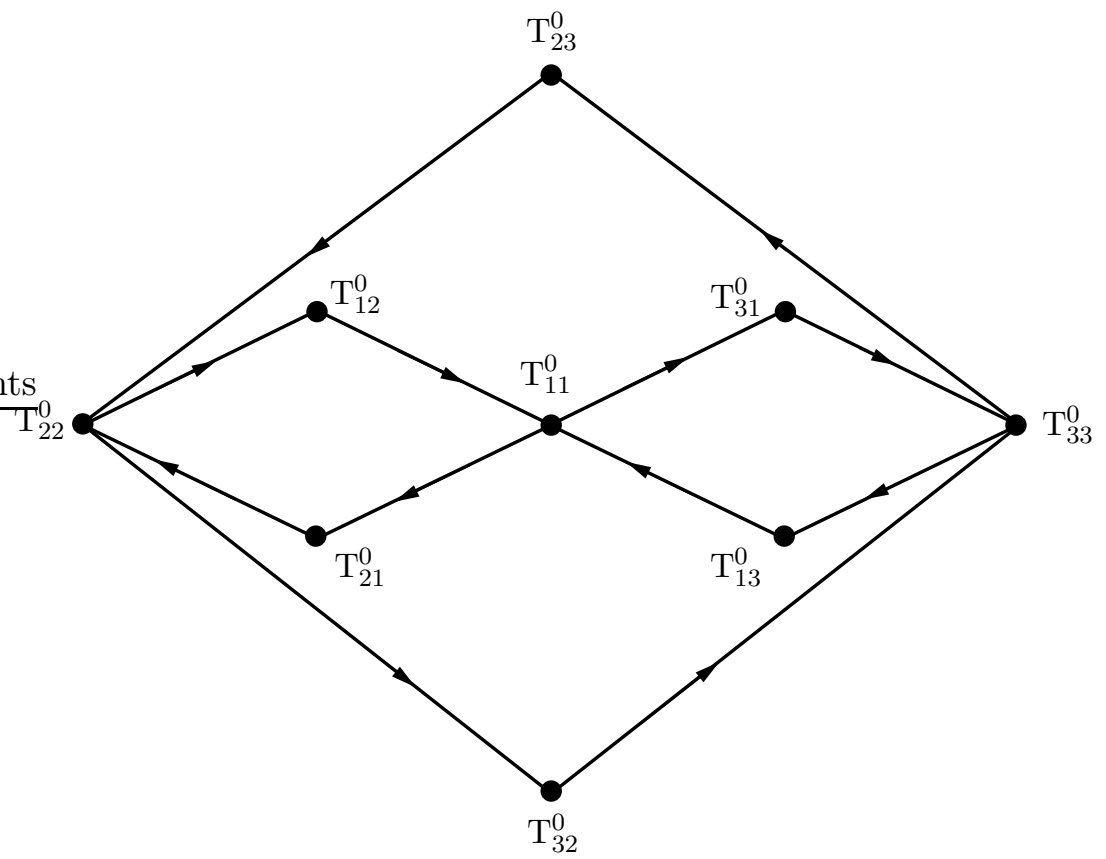

Figure 6: Schematic representation of $\mathcal{H}^{0}$.

network (or a heteroclinic subcycle thereof). This concludes the proof of the massless case of Theorem 4.3

\section{Global dynamics in the massive case}

Let $\gamma$ be an arbitrary orbit in the interior of the state space $\mathcal{X}$. The function $M_{(1)}$ is strictly monotonically increasing on $\mathcal{X}$ (and on $\mathcal{K}$ ), cf. (18) ff.; moreover, $M_{(1)}$ vanishes for $z \rightarrow 1$ and $s_{i} \rightarrow 0$ (unless $z \rightarrow 0$ simultaneously). Hence, by applying the monotonicity principle we obtain that the $\alpha$-limit set $\alpha(\gamma)$ of $\gamma$ must be located on $\mathcal{Z}^{0}$ including its boundaries.

Consider the fixed point $\mathrm{F}^{0} \in \mathcal{Z}^{0}$. By Theorem 4.2 this fixed point is a global sink on $\mathcal{Z}^{0}$. In the orthogonal direction, however, we have $\left.z^{-1} z^{\prime}\right|_{F^{0}}=2$. It follows that $\mathrm{F}^{0}$ is a hyperbolic saddle in the space $\mathcal{X}$ and that there exists exactly one orbit $\phi$ that emanates from $\mathrm{F}^{0}$ into the interior of $\mathcal{X}$. (Theorem 4.2 implies that $\phi$ converges to FS $^{1}$ as $\tau \rightarrow \infty$; thus, $\phi$ represents the unique solution of the Einstein-Vlasov equations that isotropizes toward the past and the future.)

Let $\gamma$ be different from $\phi$. Assume that $\alpha(\gamma)$ contains a point $\mathrm{P}$ of the interior of $\mathcal{Z}^{0}$; then the whole orbit through $\mathrm{P}$ and the $\omega$-limit $\omega(\mathrm{P})$ must be contained in $\alpha(\gamma)$. Theorem 4.2 implies $\omega(\mathrm{P})=\mathrm{F}^{0}$, hence $\mathrm{F}^{0} \in \alpha(\gamma)$. Since the saddle $\mathrm{F}^{0}$ is in $\alpha(\gamma)$, the unique orbit $\phi$ emanating from it is contained in $\alpha(\gamma)$ as well. Thus, ultimately, $\omega(\phi)$, i.e., a point on $\mathrm{FS}^{1}$, must be contained in $\alpha(\gamma)$; this is a contradiction, since $\mathrm{FS}^{1}$ consists of transversally hyperbolic sinks. We conclude that $\gamma$ cannot contain any $\alpha$-limit point in the interior of $\mathcal{Z}^{0}$. 
Since $\alpha(\gamma)$ must be located on the boundary on $\mathcal{Z}^{0}$, i.e., on $\mathcal{S}_{i}^{0}$ or $\mathcal{K}^{0}$, the proof can be completed in close analogy to the proof in the massless case. We thus restrict ourselves here to giving some relations that establish that the sources on $\mathcal{Z}^{0}$ generalize to sources on $\mathcal{X}$ : on $\mathrm{KC}_{i}^{0}$ we have $\left.z^{-1} z^{\prime}\right|_{\mathrm{KC}_{i}^{0}}=2\left(1+\Sigma_{i}\right)$, which is positive for all $\Sigma_{i}>-1$ and thus for $\Sigma_{i}>1$

in particular; for $\mathrm{QL}_{i}^{0}$ we obtain $\left.z^{-1} z^{\prime}\right|_{\mathrm{QL}_{i}^{0}}=4$. We note further that $\left.z^{-1} z^{\prime}\right|_{\mathrm{D}_{i}^{0}}=3$; thus $\mathrm{D}_{i}^{0}$ possesses a two-dimensional unstable manifold. (Orbits in that manifold converge to $\mathrm{FS}^{1}$.) Finally, note that along the heteroclinic cycle $\mathcal{H}_{1}^{0}: \mathrm{T}_{22}^{0} \rightarrow \mathrm{T}_{32}^{0} \rightarrow \mathrm{T}_{33}^{0} \rightarrow \mathrm{T}_{23}^{0} \rightarrow \mathrm{T}_{22}^{0}$, we obtain that $z^{-1} z^{\prime}$ equals $6 s_{2}, 2\left(1+\Sigma_{3}\right), 2\left(1+\Sigma_{2}\right), 6\left(1-s_{2}\right)$, respectively; hence $z^{-1} z^{\prime}$ is non-negative along the heteroclinic network $\mathcal{H}$.

This concludes the proof of Theorem 4.3.

\section{Concluding remarks}

In this article we have analyzed the asymptotic behaviour of solutions of the EinsteinVlasov equations with Bianchi type I symmetry. To that end we have reformulated the equations as a system of autonomous differential equations on a compact state space, which enabled us to employ powerful techniques from dynamical systems theory.

Based on the global dynamical systems analysis we have identified all possible past attractors of orbits - both in the massless and massive case. We have found that an open set of solutions converges to the Kasner circle(s); in particular, for these solutions, the rescaled matter quantity $\Omega$ satisfies $\Omega \rightarrow 0$ toward the singularity, so that "matter does not matter." However, we have seen that there exists an interesting structure that might complicate matters: there exists a heteroclinic network $\mathcal{H}^{0}$ that might be part of the past attractor set. For solutions that converge to $\mathcal{H}^{0}, \Omega$ has no limit toward the singularity, since $\Omega \neq 0$ along parts of the network $\mathcal{H}^{0}$, i.e., matter does matter for such solutions. It is not clear at the present stage whether the set of solutions converging to $\mathcal{H}^{0}$ is empty, or, if non-empty, of measure zero or not (the flow on the boundary subsets gives a hint that it might be a three-parameter set, i.e., a set of measure zero). In any case $\mathcal{H}^{0}$ will be important for the intermediate dynamical behaviour of some models, and thus there are significant differences between Bianchi type I perfect fluid models and models with Vlasov matter.

If a generic set of orbits converges to $\mathcal{H}^{0}$, this will have considerable consequences. Bianchi type I perfect fluid models play a central role in understanding the singularity of more general spatially homogeneous models [1], as well as general inhomogeneous models [6]. The importance of Bianchi type I perfect fluid models is due to Lie and source "contractions" in spatially homogeneous cosmology and the associated invariant subset and monotone function structure (which is quite similar to the hierarchical structure we have encountered in the present paper), and asymptotic silence in general inhomogeneous models 6]. Similarly, we expect that Bianchi type I Einstein-Vlasov models hold an equally prominent place as regards singularities in more general - spatially homogeneous and inhomogeneous - Einstein-Vlasov models. Hence the resolution of the problem of whether the heteroclinic network attracts generic solutions determines if Einstein-Vlasov models are generically different from general relativistic perfect fluid models in the vicinity of a 
generic singularity, or not.

In this article we have not considered a cosmological constant, $\Lambda$. The effects of a positive cosmological constant can be outlined as follows: since $\rho \rightarrow \infty$ toward the singularity, it follows that $\Lambda$ can be asymptotically neglected and hence that the singularity structure is qualitatively the same as for $\Lambda=0$. However, toward the future $\Lambda$ destabilizes FS ${ }^{1}$, which becomes a saddle, and instead solutions isotropize and asymptotically reach a de Sitter state.

We conclude with some remarks on different formulations. We have seen that the variables we have used to reformulate the equations as a dynamical system yielded multiple representations of some structures, e.g., the Kasner circle. Replacing $s_{i}$ with $E_{i}=\sqrt{g^{i i}} / H$, i.e., the Hubble-normalized spatial frame variables of [6, 12, and using $y=m^{2} H^{-2}$ instead of $z$, yields a single Kasner circle. The latter variables, however, are not bounded; indeed, they blow up toward the future in the present case. It is possible to replace the variables by bounded variables, however, variables of this type lead to differentiability difficulties toward the singularity. Issues like these made the variables we employed in this article more suitable for the kind of analysis we have performed. However, $E_{i}$-variables, or " $E_{i}$ based" variables would have been more suitable to relate the present results to a larger context; but it is not difficult to translate our results to the $E_{i}$-variables variables used in 6, 12, where the relationship between the dynamics of inhomogeneous and spatially homogeneous models was investigated and exploited.

\section{Acknowledgement}

We gratefully acknowledge the hospitality and the support of the Isaac Newton Institute for Mathematical Sciences in Cambridge, England, where part of this work was done. We also thank Alan Rendall for useful questions and comments. CU is supported by the Swedish Research Council.

\section{A Dynamical systems}

In this appendix we briefly recall some concepts from the theory of dynamical systems which we use in the article.

Consider a dynamical system defined on an invariant set $X \subseteq \mathbb{R}^{m}$. The $\omega$-limit set $\omega(x)$ $[\alpha$-limit set $\alpha(x)]$ of a point $x \in X$ is defined as the set of all accumulation points of the future [past] orbit of $x$. The simplest examples are fixed points and periodic orbits.

The monotonicity principle [1] gives information about the global asymptotic behaviour

of the dynamical system. If $M: X \rightarrow \mathbb{R}$ is a $\mathcal{C}^{1}$ function which is strictly decreasing along 
orbits in $X$, then

$$
\begin{aligned}
& \omega(x) \subseteq\left\{\xi \in \bar{X} \backslash X \mid \lim _{\zeta \rightarrow \xi} M(\zeta) \neq \sup _{X} M\right\} \\
& \alpha(x) \subseteq\left\{\xi \in \bar{X} \backslash X \mid \lim _{\zeta \rightarrow \xi} M(\zeta) \neq \inf _{X} M\right\}
\end{aligned}
$$

for all $x \in X$.

Locally in the neighbourhood of a fixed point, the flow of the dynamical system is determined by the stability features of the fixed point. If the fixed point is hyperbolic, i.e., if the linearization of the system at the fixed point is a matrix possessing eigenvalues with nonvanishing real parts, then the Hartman-Grobman theorem applies: in a neighbourhood of a hyperbolic fixed point the full nonlinear dynamical system and the linearized system are topologically equivalent. Non-hyperbolic fixed points are treated in centre manifold theory: the reduction theorem generalizes the Hartman-Grobman theorem; for further details see, e.g., [13. If a fixed point is an element of a connected fixed point set (line, surface,...) and the number of eigenvalues with zero real parts is equal to the dimension of the fixed point set, then the fixed point is called transversally hyperbolic. Application of the centre manifold reduction theorem is particularly simple in this case. (The situation is analogous in the more general case when the fixed point is an element of an a priori known invariant set that coincides with the centre manifold of the fixed point.)

\section{B FRW and LRS Symmetry $_{i}$}

In this section we discuss in detail the sets FRW and $\mathrm{LRS}_{i}$, connected with solutions exhibiting FRW or LRS geometry.

To begin with, we prove that the fixed point $\mathrm{F}^{0}$ on $z=0$ is well-defined. Since the defining equations for $\mathrm{F}^{0}$ are $w_{1}=w_{2}=w_{3}=1 / 3$, we must show that these equations indeed possess a unique solution $\left(s_{1}, s_{2}, s_{3}\right)$ for all distribution functions $f_{0}$. Setting $z=0$ in (9) implies that equations $w_{1}=w_{2}=w_{3}=1 / 3$ are equivalent to the system

$$
u:=\int f_{0}\left[s_{1} v_{1}^{2}-s_{2} v_{2}^{2}\right]\left(\sum_{k} s_{k} v_{k}^{2}\right)^{-1 / 2} d^{3} v=0
$$

and $v=0$, where $v$ is defined by replacing $\left[s_{1} v_{1}^{2}-s_{2} v_{2}^{2}\right]$ by $\left[s_{1} v_{1}^{2}-s_{3} v_{3}^{2}\right]$ in (29). On the three boundaries of the space $\left\{\left(s_{1}, s_{2}, s_{3}\right) \mid s_{i} \geq 0, \sum_{k} s_{k}=1\right\}$ the functions $u$ and $v$ are monotonic; their signs are given in Figure 7 The derivative $\partial u / \partial s_{1}$ is manifestly positive, $\partial u / \partial s_{2}$ is negative, hence grad $u$ is linearly independent of the surface normal $(1,1,1)$, and it follows that $u=$ const describes a curve for all const $\in \mathbb{R}$. The same argument applies to $v$, since $\partial v / \partial s_{1}>0$ and $\partial v / \partial s_{3}<0$. Figure 7 reveals that $u=0(v=0)$ connects the upper (right) vertex of the $\left(s_{1}, s_{2}, s_{3}\right)$-space with the opposite side. Investigating ( $\operatorname{grad} u-\lambda \operatorname{grad} v$ ) we find that the first component is manifestly positive when $\lambda \leq 2 / 3$ and negative when $\lambda \geq 3 / 2$, the second component is negative when $\lambda \leq 3$, and the third component is positive when $\lambda \geq 1 / 3$, which implies that ( $\operatorname{grad} u-\lambda \operatorname{grad} v)$ is linearly independent of the surface normal $(1,1,1)$ for all $\lambda$. It follows that all equipotential curves of the functions $u$ and $v$ intersect transversally; hence $u=0$ and $v=0$ possess a unique point of intersection, which proves the claim. 


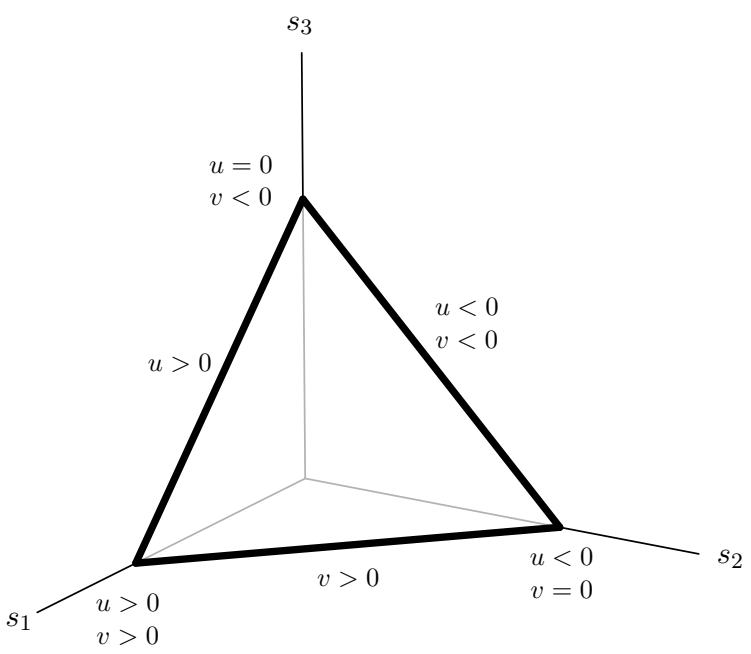

Figure 7: The functions $u$ and $v$ are monotonic along the boundaries of the space $\left\{\left(s_{1}, s_{2}, s_{3}\right) \mid s_{i} \geq 0, \sum_{k} s_{k}=1\right\}$.

By establishing existence and uniqueness of the fixed point $\mathrm{F}^{0}$ for all $f_{0}$, we have shown that for all distribution functions there exists a unique FRW solution of the massless Einstein-Vlasov equations.

The situation is different in the massive case. A FRW solution is characterized by the equations $\Sigma_{i}=0 \forall i, w_{1}=w_{2}=w_{3}=w$, since this yields $s_{i}=$ const (and a rescaling of the spatial coordinates then results in $g_{i j} \propto \delta_{i j}$.) However, for a general distribution function $f_{0}$, these equations are incompatible with the Einstein-Vlasov equations; in other words, the straight line $\Sigma_{i}=0 \forall i, w_{1}=w_{2}=w_{3}=w$ is not an orbit of the dynamical system. Hence, in the massive case, the Einstein-Vlasov equations do not admit a FRW solution for arbitrary $f_{0}$; the distribution function $f_{0}$ is required to satisfy FRW compatibility conditions, see below, in order for a FRW solution to exist.

Note, however, that for each $f_{0}$, there exists exactly one orbit that originates from $\mathrm{F}^{0}$ and ends on $\mathrm{FS}^{1}$, see Section 4, i.e., there exists a unique solution of the Einstein-Vlasov equations that isotropizes toward the past and toward the future. This anisotropic solution can be regarded as a generalized FRW solution; if $f_{0}$ is compatible with the FRW geometry, then the generalized FRW solution reduces to an ordinary FRW solution.

The treatment of the LRS case is analogous: the subset $\mathrm{LRS}_{1}$ (and, analogously, $\mathrm{LRS}_{2,3}$ ), defined through the equations $\Sigma_{2}=\Sigma_{3}, w_{2}=w_{3}$, describes solutions exhibiting LRS geometry. (For a solution on $\mathrm{LRS}_{1}$, Equation (13b) entails $s_{2}(\tau) \propto s_{3}(\tau)$; by rescaling the coordinates one can achieve $g_{22}=g_{33}$, i.e., a line element in an explicit LRS form.) However, for general $f_{0}$, the set $\mathrm{LRS}_{1}$ is not invariant under the flow of the dynamical system. Consequently, for general $f_{0}$, the Einstein-Vlasov equations do not admit solutions with LRS geometry. 
More specifically, consider

$$
\left(\Sigma_{2}-\Sigma_{3}\right)^{\prime}=-3 \Omega\left[\frac{1}{2}(1-w)\left(\Sigma_{2}-\Sigma_{3}\right)-\left(w_{2}-w_{3}\right)\right] .
$$

Hence, $\left(\Sigma_{2}-\Sigma_{3}\right)^{\prime}$ vanishes when $\Sigma_{2}=\Sigma_{3}$ and $w_{2}=w_{3}$. From (13b) and (13c) we obtain an equation for $w_{i}^{\prime}$,

$$
w_{i}^{\prime}=-2 w_{i}\left[\Sigma_{i}-\sum_{k} \Sigma_{k}\left(\frac{1}{2} w_{k}+\frac{1}{2} w_{i}^{-1} \beta_{i k}^{(0)}\right)+\frac{z}{2}\left(w_{i}^{-1} \beta_{i}^{(1)}+\beta^{(1)}\right)\right],
$$

where we have defined

$$
\beta_{i_{1} \ldots i_{k}}^{(\mathrm{m})}=\frac{(1-z)^{k} \int f_{0}\left(\Pi_{n=1}^{k} s_{i_{n}} v_{i_{n}}^{2}\right)\left[z+(1-z) \sum_{k} s_{k} v_{k}^{2}\right]^{1 / 2-k-m} d v_{1} d v_{2} d v_{3}}{\int f_{0}\left[z+(1-z) \sum_{k} s_{k} v_{k}^{2}\right]^{1 / 2} d v_{1} d v_{2} d v_{3}}
$$

note that $w_{i}=\beta_{i}^{(0)}$. Equation (31) implies

$$
\left(w_{2}-w_{3}\right)^{\prime}=-\left(\Sigma_{1}-\Sigma_{2}\right)\left(\beta_{22}^{(0)}-\beta_{33}^{(0)}\right)-z\left(\Sigma_{1}+1\right)\left(\beta_{2}^{(1)}-\beta_{3}^{(1)}\right),
$$

when $\Sigma_{2}=\Sigma_{3}$ and $w_{2}=w_{3}$. We conclude that the set $\Sigma_{2} \equiv \Sigma_{3}, w_{2} \equiv w_{3}$ is an invariant set of the dynamical system, iff $w_{2}=w_{3}$ implies $\beta_{22}^{(0)}=\beta_{33}^{(0)}$ and $\beta_{2}^{(1)}=\beta_{3}^{(1)}$. (In the massless case, only the first condition is required.) These conditions are violated for general distribution functions; for the condition to hold $f_{0}$ must be of a certain type that ensures compatibility with the LRS symmetry. This is the case, for instance, when there exist constants $a_{2}>0, a_{3}>0$, such that $f_{0}$ is invariant under the transformation $v_{2} \rightarrow\left(a_{3} / a_{2}\right) v_{3}, v_{3} \rightarrow\left(a_{2} / a_{3}\right) v_{2}$; e.g., $f_{0}=\tilde{f}_{0}\left(v_{1}, v_{2}^{2} v_{3}^{2}\right)$, or $f_{0}=\tilde{f}_{0}\left(v_{1}, a_{2}^{2} v_{2}^{2}+a_{3}^{2} v_{3}^{2}\right)$; in the latter case $w_{2}(\tau) \equiv w_{3}(\tau)$ implies $a_{3}^{2} s_{2}(\tau) \equiv a_{2}^{2} s_{3}(\tau)$.

Note finally that a distribution function $f_{0}$ is compatible with a FRW geometry, if it is compatible with all LRS symmetries. This means, that for instance $f_{0}=\tilde{f}_{0}\left(a_{1}^{2} v_{1}^{2}+a_{2}^{2} v_{2}^{2}+\right.$ $\left.a_{3}^{2} v_{3}^{2}\right)$ is compatible with the FRW symmetry and thus admits a unique FRW solution of the Einstein-Vlasov equations.

\section{Future asymptotics}

In this section we give the proof of Theorem 4.2 .

Theorem 4.2. The $\omega$-limit of every orbit in the interior of the massive state space $\mathcal{X}$ [massless state space $\mathcal{Z}^{0}$ ] is one the fixed points $\mathrm{FS}^{1}$ [the fixed point $\mathrm{F}^{0}$ ].

Proof. Consider first the state space $\mathcal{Z}^{0}$ of massless particles and the associated system (19). The function $M_{(2)}$, cf. (20) ff., is well-defined and monotonically decreasing everywhere except for at the fixed point $\mathrm{F}^{0}$, where it has a global minimum. On the boundaries $\mathcal{S}_{i}^{0}$ (given by $s_{i}=0$ ) and $\mathcal{K}^{0}\left(\Sigma^{2}=1\right.$ ) of the state space $\mathcal{Z}^{0}$, the function $M_{(2)}$ is infinite. Therefore, application of the monotonicity principle yields that the $\omega$-limit of every orbit must be the fixed point $\mathrm{F}^{0}$. 
In the massive case consider (13a) in the form

$$
\Sigma_{i}^{\prime}=-3 \Omega\left[\frac{1}{2}(1-w)\left(1+\Sigma_{i}\right)-\frac{1}{2}(1-3 w)-w_{i}\right] .
$$

The r.h.s. is positive when $\Sigma_{i} \leq-1$ and $z>0(w<1 / 3)$. This implies that the hyperplanes $\Sigma_{i}=-1$ constitute semipermeable membranes in the state space $\mathcal{X}$, whereby the "triangle" $\left(\Sigma_{1}>-1\right) \cap\left(\Sigma_{2}>-1\right) \cap\left(\Sigma_{3}>-1\right)$ becomes a future invariant subset of the flow (13).

The first part of the proof is to show that every orbit enters the triangle at some time $\tau_{e}$ (and consequently remains inside for all later times).

Assume that there exists an orbit with $\Sigma_{i}(\tau) \leq-1$ for all $\tau$ (for some $i$ ). From (13b) we infer that

$$
s_{i}^{\prime}=-2 s_{i}\left[s_{j}\left(\Sigma_{i}-\Sigma_{j}\right)+s_{k}\left(\Sigma_{i}-\Sigma_{k}\right)\right]>0
$$

if $\Sigma_{i}<-1$, and that $s_{i}^{\prime} \geq 0$ if $\Sigma_{i}=-1$; hence $s_{i}(\tau) \geq s_{i}\left(\tau_{0}\right)=$ const $>0$ for all $\tau \in\left[\tau_{0}, \infty\right)$. From (16) we obtain

$$
\begin{aligned}
\left.\frac{1}{3} \Omega^{-1} \Omega^{\prime}\right|_{\Omega=0} & =1-\frac{1}{3} w_{i}\left(1+\Sigma_{i}\right)-\frac{1}{3} w_{j}\left(1+\Sigma_{j}\right)-\frac{1}{3} w_{k}\left(1+\Sigma_{k}\right) \geq \\
& \geq 1-w_{j}-w_{k}=(1-3 w)+w_{i} \geq \mathrm{const}>0,
\end{aligned}
$$

since $s_{i} \geq$ const $>0$. Consequently, $\Omega(\tau) \geq$ const $>0$ for all $\tau \in\left[\tau_{0}, \infty\right)$. It follows from (34) that

$$
\Sigma_{i}^{\prime} \geq \text { const }>0
$$

for all $\tau \in\left[\tau_{0}, \infty\right)$ by the same argument. This is in contradiction to the assumption $\Sigma_{i} \leq-1$ for all $\tau$.

Thus, in the second part of the proof, we can consider an arbitrary orbit $\gamma$ and assume, without loss of generality, that $\gamma(\tau)$ lies in the $\Sigma$-triangle for all $\tau \in\left[\tau_{e}, \infty\right)$. Equation (13c) leads to

$$
z^{\prime}=2 z(1-z) \sum_{n} s_{n}\left(1+\Sigma_{n}\right) \geq 0
$$

for all $\tau \in\left[\tau_{e}, \infty\right)$, hence $z(\tau) \geq z\left(\tau_{e}\right)>0$ for all $\tau \in\left[\tau_{e}, \infty\right)$.

We define the function $N$ by

$$
N=\left(1+\Sigma_{1}\right)\left(1+\Sigma_{2}\right)\left(1+\Sigma_{3}\right) .
$$

The derivative can be estimated by

$$
N^{\prime} \geq 3 \Omega N\left[-\frac{3}{2}(1-w)+\frac{1}{2} \sum_{n} \frac{1-3 w}{1+\Sigma_{n}}\right] .
$$

Since $w(\tau) \leq$ const $<1 / 3$ (because $z(\tau) \geq$ const $>0$ ), $N^{\prime}$ is positive when at least one of the $\Sigma_{i}$ is sufficiently small, i.e., when $N$ itself is small (a detailed analysis shows that $\left.N^{\prime} \geq 3 \Omega N\left[-(3 / 2)(1-w)+\sqrt{3}(1-3 w) N^{-1 / 2}\right]\right)$. We conclude that there exists a positive constant $N_{0}$ such that $N(\tau) \geq N_{0}$ for all $\tau \in\left[\tau_{e}, \infty\right)$. This in turn implies that there exists $\nu>0$ such that $\Sigma_{i}(\tau) \geq-1+\nu$ for all $i$ for all $\tau \in\left[\tau_{e}, \infty\right)$, whereby $z^{\prime} \geq 2 z(1-z) \nu$ for all $\tau \in\left[\tau_{e}, \infty\right)$. 
It follows that the $\omega$-limit of $\gamma$ must lie on $z=1$, i.e., on $\mathcal{Z}^{1}$. Taking into account the simple structure of the flow on $\mathcal{Z}^{1}$, characterized by $\Omega^{\prime}=3(1-\Omega) \Omega$, we conclude that the fixed points $\mathrm{FS}^{1}$ given by $\Sigma_{1}=\Sigma_{2}=\Sigma_{3}=0$ are the only possible $\omega$-limits.

Remark. In order to demonstrate the versatility of the dynamical systems methods, we have chosen here to prove Theorem 4.2 by using techniques that are slightly different from those employed in Section 4 (which exploit the monotonicity principle). However, it is straightforward (in fact, even simpler) to give a proof by making use of the hierarchy of monotone functions. Indeed, the function $M_{(1)}$ ensures that the $\omega$-limit of every orbit lies on $\mathcal{Z}^{1}$ or $\mathcal{S}_{i}$; modulo some subtleties, we can exclude that $\mathcal{S}_{i}$ is attractive by using the monotone function $M_{(3)}$ and the local properties of the fixed points.

\section{The spaces $\mathcal{S}_{i}^{0}$ - interpretation of solutions}

The flow on the boundary subsets $\mathcal{S}_{i}^{0}$ is of fundamental importance in the analysis of the global dynamics of the state space, see Section 4.2. Note that except for $\mathrm{F}^{0}$ all attractors $\left(\mathrm{D}_{i}^{0}, \mathrm{QL}_{i}^{0}, \mathrm{KC}_{i}^{0}\right.$, and the heteroclinic network) lie on $\mathcal{S}_{i}^{0}$. For a depiction of the flow on $\mathcal{S}_{1}^{0}$ see Figure 3. In the following we show that orbits on $\mathcal{S}_{1}^{0}$ represent solutions of the Einstein-Vlasov system that are associated with a special class of distribution functions. Furthermore, we investigate in detail solutions that converge to the subcycle $\mathcal{H}_{1}^{0}$ of the heteroclinic network.

Consider a distribution function $f_{0}$ of the form

$$
f_{0}\left(v_{1}, v_{2}, v_{3}\right)=\delta\left(v_{1}\right) f_{0}^{\mathrm{red}}\left(v_{2}, v_{3}\right),
$$

where $f_{0}^{\text {red }}\left(v_{2}, v_{3}\right)$ is even in $v_{2}$ and $v_{3}$. In the case of massless particles, $m=0$ (and $z=0$ respectively), we obtain

$$
w_{1}=0, \quad w_{j}=\frac{g^{j j} \int f_{0}^{\text {red }} v_{j}^{2}\left[g^{22} v_{2}^{2}+g^{33} v_{3}^{2}\right]^{-1 / 2} d v_{1} d v_{2} d v_{3}}{\int f_{0}^{\text {red }}\left[g^{22} v_{2}^{2}+g^{33} v_{3}^{2}\right]^{1 / 2} d v_{1} d v_{2} d v_{3}}(j=2,3),
$$

where $g^{22}$ and $g^{33}$ can be replaced by $s_{2}$ and $s_{3}$, if desired. In the unbounded variables $g^{i i}$ the equations read

$$
\begin{array}{ll}
\Sigma_{1}^{\prime}=-\Omega\left[1+\Sigma_{1}\right], & \left(g^{11}\right)^{\prime}=-2 g^{11}\left(1+\Sigma_{1}\right) \\
\Sigma_{j}^{\prime}=-\Omega\left[1+\Sigma_{j}-3 w_{j}\right], & \left(g^{j j}\right)^{\prime}=-2 g^{j j}\left(1+\Sigma_{j}\right)
\end{array}
$$

cf. the remark at the end of Section 2. In particular we note that the equation for $g^{11}$ decouples; hence the full dynamics is represented by a reduced system in the variables $\left(\Sigma_{1}, \Sigma_{2}, \Sigma_{3}, g^{22}, g^{33}\right)$, which coincides with the system (43) on the invariant subset $g^{11}=0$. In analogy to the definitions (7a) we set

$$
s_{1}=0, \quad s_{2}=\frac{g^{22}}{g^{22}+g^{33}}, \quad s_{3}=\frac{g^{33}}{g^{22}+g^{33}},
$$


so that $s_{2}+s_{3}=1$. This results in the dynamical system

$$
\begin{aligned}
& \Sigma_{1}^{\prime}=-\Omega\left[1+\Sigma_{1}\right], \quad s_{1} \equiv 0 \\
& \Sigma_{j}^{\prime}=-\Omega\left[1+\Sigma_{j}-3 w_{j}\right], \quad s_{j}^{\prime}=-2 s_{j}\left[\Sigma_{j}-\left(s_{2} \Sigma_{2}+s_{3} \Sigma_{3}\right)\right] \quad(j=2,3) .
\end{aligned}
$$

This system (45) coincides with the dynamical system (13) induced on $\mathcal{S}_{1}^{0}$ (which is obtained by setting $z=0$, thus $w=1 / 3$, and $s_{1}=0$ in (13)).

Our considerations show that the flow on $\mathcal{S}_{1}^{0}$ possesses a direct physical interpretation: orbits on $\mathcal{S}_{1}^{0}$ represent solutions of the massless Einstein-Vlasov system of Bianchi type I with a "distributional" distribution function of the type (41). Note that the system (45) on $\mathcal{S}_{1}^{0}$ must be supplemented by the decoupled equations (11b) and $\left(g^{11}\right)^{\prime}=-2 g^{11}\left(1+\Sigma_{1}\right)$ in order to construct the actual solution from an orbit in $\mathcal{S}_{1}^{0}$.

Two structures in $\mathcal{S}_{1}^{0}$ are of particular interest: the fixed point $\mathrm{D}_{1}^{0}$ and the heteroclinic cycle $\mathcal{H}_{1}^{0}$, see Figure 3 The fixed point $\mathrm{D}_{1}^{0}$ represents an LRS solution (associated with a distributional $f_{0}$ ); it is straightforward to show that the metric is of the form

$$
g_{11}=\text { const }, \quad g_{22} \propto t^{4 / 3}, \quad g_{33} \propto t^{4 / 3},
$$

and $H=(4 / 9) t^{-1}$.

The orbit $\mathrm{T}_{22}^{0} \rightarrow \mathrm{T}_{32}^{0}$, which is part of $\mathcal{H}_{1}^{0}$, corresponds to a solution

$$
g_{11}=g_{11}^{0}, \quad g_{22}=g_{22}^{0}\left(3 H_{0} t\right)^{2}, \quad g_{33}=g_{33}^{0} ;
$$

here, $H=(3 t)^{-1} ; H_{0}$ is a characteristic value of $H$. For the orbit $\mathrm{T}_{33}^{0} \rightarrow \mathrm{T}_{23}^{0}$ the result is analogous with $g_{22}$ and $g_{33}$ interchanged. A more extensive computation shows that the orbit $\mathrm{T}_{32}^{0} \rightarrow \mathrm{T}_{33}^{0}$ leads to

$$
g_{11}=g_{11}^{0}, \quad g_{22}=g_{22}^{0}\left[\log \left(1+3 H_{0} t\right)\right]^{2}, \quad g_{33}=g_{33}^{0}\left(1+3 H_{0} t\right)^{2},
$$

together with $H=H_{0}\left(1+3 H_{0} t\right)^{-1}\left(1+\left[\log \left(1+3 H_{0} t\right)\right]^{-1}\right)$. (Note that $3 H t$ is always close to 1 and approaches 1 for $t \rightarrow 0$ and $t \rightarrow \infty$.) The result for the orbit $\mathrm{T}_{23}^{0} \rightarrow \mathrm{T}_{22}^{0}$ is analogous with $g_{22}$ and $g_{33}$ interchanged.

Now consider an orbit converging to the heteroclinic cycle as $\tau \rightarrow-\infty$, i.e., $t \searrow 0$. Since the orbit alternates between episodes where it is close to one of the four heteroclinic orbits, we obtain a solution with alternating episodes of characteristic behaviour of the type (47) and (48); transitions between the episodes correspond to the orbit being close to the fixed points.

Let $t^{(n)}$ denote a monotone sequence of times such that the solution is in episode $(n)$ at time $t^{(n)}$ (i.e., the orbit is close to one of the four heteroclinic orbits and far from the fixed points); $t^{(n)} \searrow 0$ as $n \rightarrow \infty$. Since $3 H t \approx 1$ as $t \searrow 0$, the sequence $t^{(n)}$ gives rise to a sequence $H^{(n)}$ defined by $3 H^{(n)} t^{(n)}=1$. During episode $(n)$ the solution exhibits a characteristic behaviour of the type (47) or (48) with $H_{0}=H^{(n)}$ (and $g_{22}^{0}=g_{22}^{(n)}$, $\left.g_{33}^{0}=g_{33}^{(n)}\right)$. A transition from one episode to another involves a matching of the constants. Example. Suppose that the orbit is close to the heteroclinic orbit $\mathrm{T}_{32}^{0} \rightarrow \mathrm{T}_{33}^{0}$ in episode $(n)$. We obtain a behaviour of the type (48) with $H_{0}=H^{(n)}$. As $H^{(n)} t$ gets small we see 
that $g_{22} \approx g_{22}^{(n)}\left(3 H^{(n)} t\right)^{2}, g_{33} \approx g_{33}^{(n)}$. The next (as $t \searrow 0$ ) episode corresponds to the orbit running close to $\mathrm{T}_{22}^{0} \rightarrow \mathrm{T}_{32}^{0}$; the behaviour of the solution is (47) with $g_{22}^{(n+1)}, g_{33}^{(n+1)}$, and $H_{0}=H^{(n+1)}$. The transition between the episodes $(n)$ and $(n+1)$ is thus straightforward: $g_{22}^{(n+1)}\left(H^{(n+1)}\right)^{2}=g_{22}^{(n)}\left(H^{(n)}\right)^{2}$ and $g_{33}^{(n+1)}=g_{33}^{(n)}$. Matching episodes $(n+1)$ and $(n+2)$ is slightly more involved. The orbit is close to the heteroclinic orbit $\mathrm{T}_{23}^{0} \rightarrow \mathrm{T}_{22}^{0}$ in episode $(n+2)$, where

$$
g_{11}=g_{11}^{0}, \quad g_{22}=g_{22}^{(n+2)}\left(1+3 H^{(n+2)} t\right)^{2}, \quad g_{33}=g_{33}^{(n+2)}\left[\log \left(1+3 H^{(n+2)} t\right)\right]^{2} .
$$

Close to transition time, when $H^{(n+2)} t$ is large, we get $g_{22}=g_{22}^{(n+2)}\left(3 H^{(n+2)} t\right)^{2}$ and $g_{33}=g_{33}^{(n+2)}\left(\log 3 H^{(n+2)} t\right)^{2}$. The transition between episode $(n+1)$ and $(n+2)$ thus involves that $g_{33}$ begins to decay logarithmically from having been approximately constant.

\section{References}

[1] J. Wainwright and G. F. R. Ellis. Dynamical systems in cosmology. Cambridge University Press, Cambridge, 1997.

[2] A. D. Rendall. The initial singularity in solutions of the Einstein-Vlasov system of Bianchi type I. J. Math. Phys. 37438 (1996).

[3] A. D. Rendall, and K. P. Tod. Dynamics of spatially homogeneous solutions of the Einstein-Vlasov equations which are locally rotationally symmetric. Class. Quantum Grav. 161705 (1999).

[4] A. D. Rendall, and C. Uggla. Dynamics of spatially homogeneous locally rotationally symmetric solutions of the Einstein-Vlasov equations. Class. Quantum Grav. 174697 (2000).

[5] A. D. Rendall. Cosmological models and centre manifold theory. Gen. Rel. Grav. 34 1277 (2002).

[6] C. Uggla, H. van Elst, J. Wainwright, and G. F. R. Ellis. The past attractor in inhomogeneous cosmology. Phys. Rev. D 68103502 (2003).

[7] C. W. Misner, K. S. Thorne and J. A. Wheeler. Gravitation. W. H. Freeman and Company, San Francisco, 1973.

[8] H. Andréasson. The Einstein-Vlasov system/Kinetic theory. Living Rev. Relativity 8 lrr-2005-2 (2005) Online journal article (cited on 30/06/2005), http://www.livingreviews.org/llr-2005-2.

[9] A. D. Rendall. The Einstein-Vlasov system. In P. T. Chruściel and H. Friedrich, editors, The Einstein Equations and the Large Scale Behaviour of Gravitational Fields, Birkhäuser 235 (2004).

[10] R. Maartens and S. D. Maharaj. Collision-free Gases in Bianchi space-times. Gen. Rel. Grav. 22595 (1990). 
[11] P. Ashwin and M. Field. Heteroclinic Networks in Coupled Cell Systems. Arch. Rational Mech. Anal. 148107 (1999).

[12] N. Röhr, and C. Uggla. Conformal regularization of Einstein's field equations. Class. Quantum Grav. 223775 (2005).

[13] J. D. Crawford. Introduction to bifurcation theory. Rev. Mod. Phys. 63-4 991 (1991). 